\title{
ETV1 directs androgen metabolism and confers aggressive prostate cancer in targeted mice and patients
}

\author{
Esther Baena, ${ }^{1,2}$ Zhen Shao, ${ }^{1,2,3,9}$ Douglas E. Linn, ${ }^{4,9}$ Kimberly Glass, ${ }^{3}$ Melanie J. Hamblen, ${ }^{1,2,5}$ \\ Yuko Fujiwara, ${ }^{1,2,5}$ Jonghwan Kim, ${ }^{1,2}$ Minh Nguyen, ${ }^{1,2}$ Xin Zhang, ${ }^{4}$ Frank J. Godinho, ${ }^{1,2,5}$ \\ Roderick T. Bronson, ${ }^{6}$ Lorelei A. Mucci, ${ }^{7}$ Massimo Loda, ${ }^{8}$ Guo-Cheng Yuan, ${ }^{3}$ Stuart H. Orkin, ${ }^{1,2,5,10}$ \\ and $\mathrm{Zhe} \mathrm{Li}^{1,2,4,10}$ \\ ${ }^{1}$ Division of Hematology and Oncology, Boston Children's Hospital, Boston, Massachusetts 02115, USA; ${ }^{2}$ Department of \\ Pediatric Oncology, Dana-Farber Cancer Institute, Harvard Medical School, Boston, Massachusetts 02115, USA; ${ }^{3}$ Department of \\ Biostatistics and Computational Biology, Dana-Farber Cancer Institute, Harvard School of Public Health, Boston, Massachusetts \\ 02115, USA; ${ }^{4}$ Division of Genetics, Brigham and Women's Hospital, Boston, Massachusetts 02115, USA; ${ }^{5}$ Howard Hughes \\ Medical Institute, Boston, Massachusetts 02115, USA; ${ }^{6}$ Pathology, Harvard Medical School, Boston, Massachusetts 02115, USA; \\ ${ }^{7}$ Department of Epidemiology, Harvard School of Public Health, Brigham and Women's Hospital, Boston, Massachusetts 02115, \\ USA; ${ }^{8}$ Center for Molecular Oncologic Pathology, Dana-Farber Cancer Institute, Brigham and Women's Hospital, Boston, \\ Massachusetts 02115, USA
}

Distinguishing aggressive from indolent disease and developing effective therapy for advanced disease are the major challenges in prostate cancer research. Chromosomal rearrangements involving ETS transcription factors, such as ERG and ETV1, occur frequently in prostate cancer. How they contribute to tumorigenesis and whether they play similar or distinct in vivo roles remain elusive. Here we show that in mice with ERG or ETV1 targeted to the endogenous Tmprss2 locus, either factor cooperated with loss of a single copy of Pten, leading to localized cancer, but only ETV1 appeared to support development of invasive adenocarcinoma under the background of full Pten loss. Mechanistic studies demonstrated that ERG and ETV1 control a common transcriptional network but largely in an opposing fashion. In particular, while ERG negatively regulates the androgen receptor (AR) transcriptional program, ETV1 cooperates with AR signaling by favoring activation of the AR transcriptional program. Furthermore, we found that ETV1 expression, but not that of ERG, promotes autonomous testosterone production. Last, we confirmed the association of an ETV1 expression signature with aggressive disease and poorer outcome in patient data. The distinct biology of ETV1-associated prostate cancer suggests that this disease class may require new therapies directed to underlying programs controlled by ETV1.

[Keywords: ERG; ETS transcription factor; ETV1; Pten; androgen receptor; metabolism]

Supplemental material is available for this article.

Received November 27, 2012; revised version accepted February 20, 2013.

Prostate cancer is a heterogeneous disease. Recent studies show little benefit from prostate-specific antigen (PSA) screening and radical prostatectomy for men with lowerrisk disease (Wilt et al. 2012). A central challenge in management is identification of those men with prostate cancer whose disease will eventually progress to the lethal castration-resistant stage. Understanding molecular events leading to castration-resistant prostate cancer (CRPC) is

\footnotetext{
${ }^{9}$ These authors contributed equally to this work.

${ }^{10}$ Corresponding authors

E-mail stuart_orkin@dfci.harvard.edu

E-mail zli4@rics.bwh.harvard.edu

Article is online at http://www.genesdev.org/cgi/doi/10.1101/gad.211011.112.

Freely available online through the Genes \& Development Open Access option.
}

critical for the development of improved therapies for such patients.

Chromosomal rearrangements involving genes encoding ETS transcription factors (notably, ERG and ETV1) are found in $\sim 50 \%$ of human prostate cancer cases and likely constitute the most frequent gene rearrangements in human malignancies (Tomlins et al. 2005; Gopalan et al. 2009). The translocations place the coding regions of $E R G$ or ETV1 under the control of androgen-responsive promoters, such as TMPRSS2, thereby activating expression in response to androgens. TMPRSS2 has been reported as the principal 5' fusion partner of $E R G$, whereas more heterogeneous 5' fusion partners, such as TMPRSS2, SCL45A3, or ACSL3, have been described for ETV1 (Tomlins et al. 2007; Attard et al. 2008b). The majority of these 5' fusion 
partners are also androgen-responsive genes. As ETS proteins, ERG and ETV1 are involved in regulation of cell growth, proliferation, differentiation, and apoptosis through activation or repression of target genes (Oikawa and Yamada 2003). Although functional overlap among different members of the ETS family exists, individual ETS factors also serve distinct roles. Thus, the expression pattern of ETS members through development varies, along with their repertoire of target genes, biological processes regulated, and oncogenic potentials (Seth and Watson 2005; Kunderfranco et al. 2010; Wei et al. 2010; Hollenhorst et al. 2011).

Clinical studies of the prevalence and prognostic significance of ETS fusions in prostate cancer have yielded discrepant results, possibly related to differences in the genetics of the evaluated populations and diversity in methods used. Several studies suggest that ETS fusions are associated with a worse prognosis (Demichelis et al. 2007; Nam et al. 2007; Attard et al. 2008a), whereas others have failed to confirm the correlation (Gopalan et al. 2009; Hermans et al. 2009; Minner et al. 2011). Cases with ETS fusions are generally grouped together for patient stratification. However, considering all ETS translocations as a single entity risks obscuring possible differences in the contribution of each to disease outcome. For example, effects of TMPRSS2-ERG, the most common translocation, may bias findings of aggregate studies. Studies to date have not specifically addressed the biology of individual ETS fusions and their associated outcomes.

In this study, we used knock-in mouse modeling and comprehensive genome-wide approaches to characterize the functional specificities of ERG and ETV1 in prostate cancer. Our data indicate that ERG and ETV1 regulate a common set of genes, such as androgen receptor (AR) target genes, but in an opposing direction. In particular, ETV1, but not ERG, up-regulates expression of AR target genes as well as genes involved in steroid biosynthesis and metabolism. This ETV1-driven oncogenic program predisposes prostate cells for cooperation with other oncogenic events, such as PTEN loss, leading to more aggressive disease in murine models and human patients. Our findings further establish different biological subtypes of human prostate cancer based on distinct ETS factor-driven signatures.

\section{Results}

Tmprss2-ETV1 cooperates with total Pten loss, leading to invasive adenocarcinoma

As a step toward defining the roles of ETS fusions in prostate cancer, several groups have generated transgenic mouse strains that express ERG or ETV1 ectopically under the control of the Probasin $(\mathrm{Pb})$ promoter $(\mathrm{Pb}-E R G$ or Pb-ETV1) (Tomlins et al. 2007, 2008; Klezovitch et al. 2008; Carver et al. 2009; King et al. 2009; Shin et al. 2009). Interpretation of results has varied. Prostatic intraepithelial neoplasia (PIN)-like lesions have been described in prostates of $P b-E R G$ and $P b-E T V 1$ transgenic males
(Tomlins et al. 2007, 2008; Klezovitch et al. 2008; Shin et al. 2009). However, others have reported that $P b-E R G$ transgenic males are normal (Carver et al. 2009; King et al. 2009). Discrepant findings may be related to mouse strain differences, to different transgene integration sites, or in the precise portions of the ETS cDNAs that were expressed. We reasoned that mice engineered to express ETS factors from an endogenous promoter in the proper chromosomal configuration might provide a more relevant biological context. Moreover, prior transgenic models cannot address potential contributions of haploinsufficiency or loss of genes deleted between TMPRSS2 and ERG to prostate tumorigenesis, such as occurs in patients with a TMPRSS2-ERG fusion generated through an interstitial deletion of chromosome 21 .

We engineered knock-in mouse models to recapitulate TMPRSS2-ETS fusions /with or without the interstitial deletion) in prostate cancer. We used two strategies. In the first strategy, we knocked in $\mathrm{N}$ terminus-truncated human ERG or ETV1 cDNA, together with an ires-GFP cassette, into exon 2 of the mouse Tmprss 2 locus (referred to as T-ERG or T-ETV1 hereafter), which shares $\sim 80 \%$ homology as well as at least two conserved AR-binding sites with those of the human TMPRSS2 (Fig. 1A; Supplemental Fig. S1; Jacquinet et al. 2000). The resultant fusion transcripts recapitulate the TMPRSS2-ERGa or TMPRSS2-ETV1a fusions in patients (Tomlins et al. 2005). In the second strategy, we used sequential gene targeting to introduce loxP sites into the Tmprss2 and Erg loci on the same chromosome (Fig. 1A; Supplemental Fig. $\mathrm{S} 2 \mathrm{~A}, \mathrm{~B})$. Cre-mediated recombination deletes the $\sim 3-\mathrm{Mb}$ intragenic region and generates the Tmprss2-Erg fusion gene (Supplemental Fig. S2C,D), which approximates the TMPRSS2-ERGa fusion subtype (Tomlins et al. 2005). Since most genes in this interstitial region are syntenic between humans and mice (Supplemental Fig. S2E), this unique knock-in model also permits assessment of the contribution of the interstitial deletion to prostate cancer development (referred to as T-3Mb-Erg or T- $\Delta$-Erg before or after Cre-mediated excision of the interstitial region, respectively) (Fig. 1A). In all three knock-in alleles (i.e., T-ETV1, T-ERG, and T- $\Delta$-Erg) we confirmed expression of their corresponding fusion transcripts in prostates (Fig. 1B). By immunohistochemistry (IHC), we detected moderate expression of Erg protein in the knock-in prostates (Fig. 1C). Despite multiple efforts, we were unable to validate an antibody that faithfully detects ETV1 protein by IHC. Therefore, we used IHC staining for GFP as a surrogate for ETV1 expression, as the knock-in GFP reporter is under the same transcriptional control (Fig. 1A). With this approach, we detected robust GFP (ETV1) expression in prostate epithelial cells but not in stromal cells (Fig. 1D). In all three knock-in strains, prostates appeared largely normal, and we did not observe prostatic intraepithelial neoplasia (PIN) lesions or cancer (Fig. 1E). However, in a portion of T-ETV1 males (four out of 11), in particular those at old ages ( $\geq 18 \mathrm{mo}$; three out of three), we observed varying degrees of inflammation (Fig. 1E). In addition, pathological analysis in several exceptional $T-\Delta$-Erg males (four out of 21 but in none of the T-ERG 
A

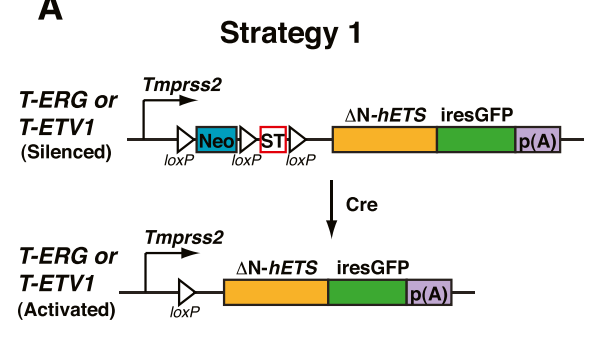

C

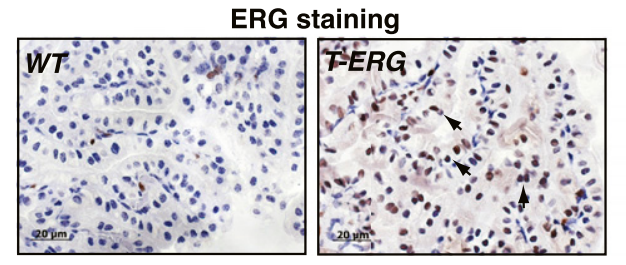

D

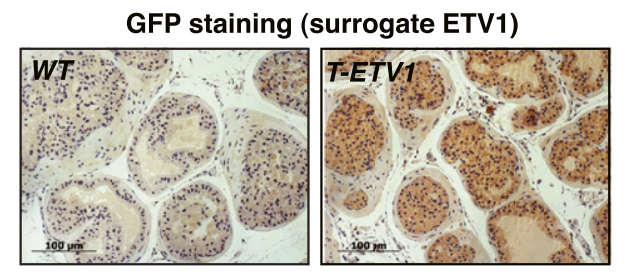

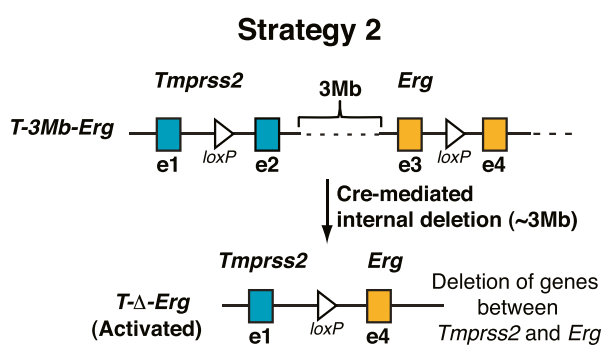

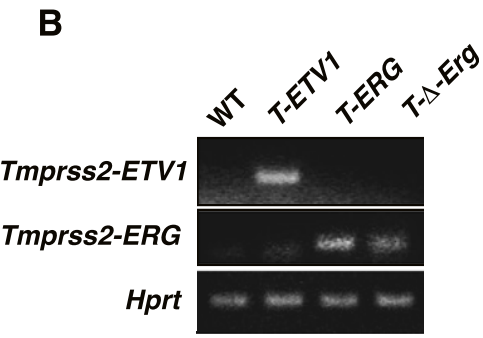

E

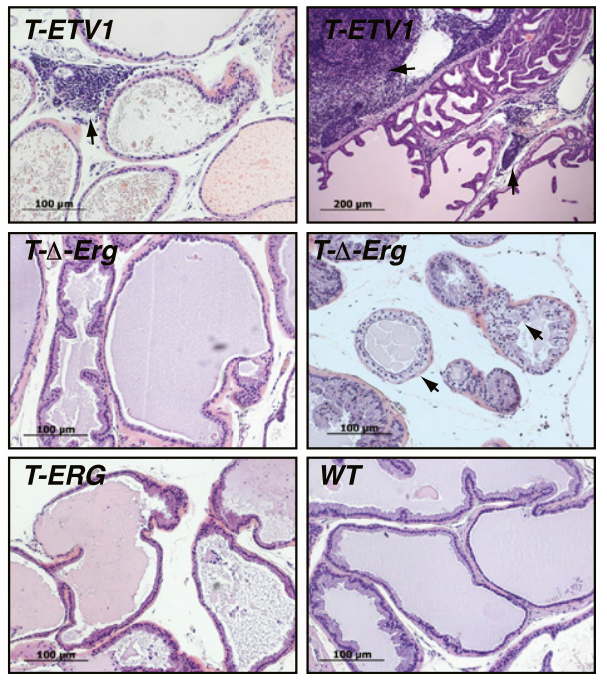

Figure 1. Tmprss2-ERG (with or without interstitial deletion) and Tmprss2-ETV1 expression are insufficient to initiate prostate tumorigenesis. (A) Targeting strategies for engineering Tmprss2-ERG and Tmprss2-ETV1 knock-ins. Strategy 1 is based on direct knockin of $\mathrm{N}$ terminus-truncated human ERG or ETV1 cDNA ( $\triangle \mathrm{N}-h E T S)$ into the murine Tmprss2 locus. Strategy 2 is based on the introduction of loxP sites to murine Tmprss2 and Erg loci by sequential gene targeting in mouse embryonic stem cells so that the 3-Mb interstitial region can be deleted by Cre-mediated recombination and meanwhile generate the Tmprss2-Erg gene fusion. Details of gene targeting are shown in Supplemental Figures S1 and S2. (B) RT-PCR showing expression of the Tmprss2-ETV1 fusion transcripts in T-ETV1 knock-in prostates and expression of the Tmprss2-ERG fusion transcripts in T-ERG and T- $\Delta$-Erg knock-in prostates but not in wild-type (WT) prostates. (C) IHC staining showing moderate ERG expression (arrows) in the anterior lobes of a T-ERG knock-in male but not in the wild-type male. $(D)$ IHC staining showing homogeneous GFP expression (as surrogate for ETV1) in the anterior lobes of a T-ETV1 knock-in male but not in the wild-type male. (E) Hemotoxylin and eosin (H\&E) staining showing normal prostate histology from all three knock-ins (showing ventral lobes except those of T-ETV1). Arrows in T-ETV1 pictures indicate inflammation in T-ETV1 knock-in males ([left] slight inflammation in the lateral lobe of a young knock-in male; [right] extensive inflammation in the anterior lobe of a 30-mo-old knock-in male). (Right) Arrows in the T- $\Delta$-Erg picture indicate abnormal-looking (lightly stained "foamy"-looking cytoplasm, randomly distributed nuclei) prostate cells,observed in four out of 21 of T- $\Delta$-Erg males. Bars, $100 \mu \mathrm{m}(200 \mu \mathrm{m}$ in top right picture). All animals analyzed in $C-E$ were $\sim 10$ mo of age unless otherwise indicated.

males) revealed some hyperplasia and foci of cells with lightly stained cytoplasm and loss of polarity (Fig. 1E). Despite these minor phenotypes, we conclude that expression of ERG or ETV1 from the endogenous Tmprss2 promoter, even in the presence of the interstitial deletion (for Erg fusion), is insufficient to initiate prostate tumorigenesis.

Overexpression of ERG or ETV1 from the $P b$ promoter or through lentiviral transduction in prostate cells has been previously reported to cooperate with activation of the PI3K pathway to drive a more aggressive prostate cancer phenotype (Carver et al. 2009; King et al. 2009; Zong et al. 2009). To determine whether this is also the case when ETV1 or ERG is expressed from the endogenous Tmprss2 promoter, we bred mice containing the knock-in alleles with $\mathrm{Pten}^{+/-}$mice. We found that within the time frame monitored (up to 15 mo of age), prostates of all aged T-ETV1;Pten ${ }^{+/}$, T-ERG;Pten ${ }^{+-}$, and T- $\Delta-$ Erg; $_{\text {Pten }}{ }^{+/}$double-mutant males developed PIN lesions that stain positive for phosphorylated AKT (pAKT), whereas prostates of Pten $^{+/-}$-alone mice appeared largely normal (Fig. 2A; Supplemental Fig. S3). In the above cohort, PIN lesions from double-mutant males maintained relatively uniform and high levels of ETV1 (GFP) or ERG expression (Fig. 2B); this is particularly notable for ERG, as in the prostates of $E R G$ knock-in alone, ERG expression was 
A

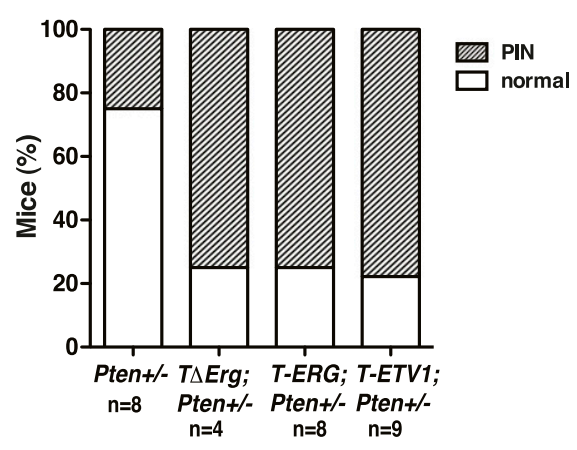

C

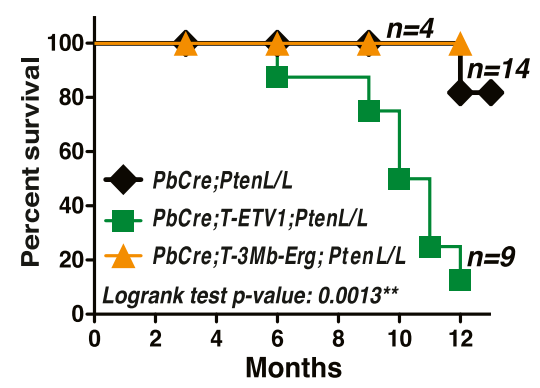

B
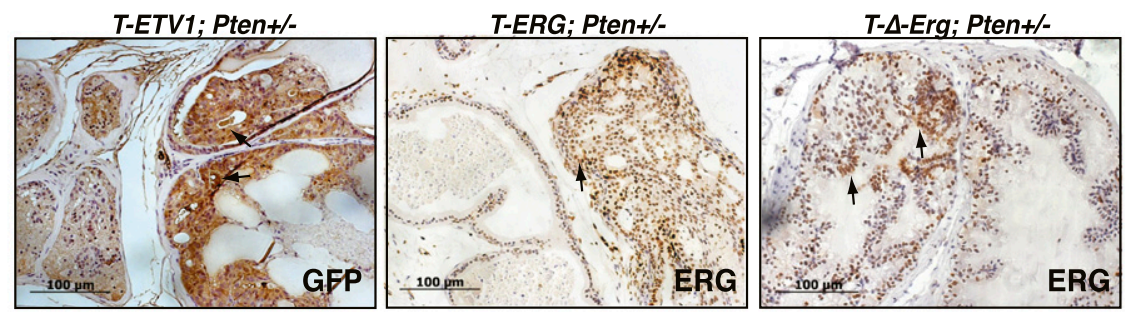

D
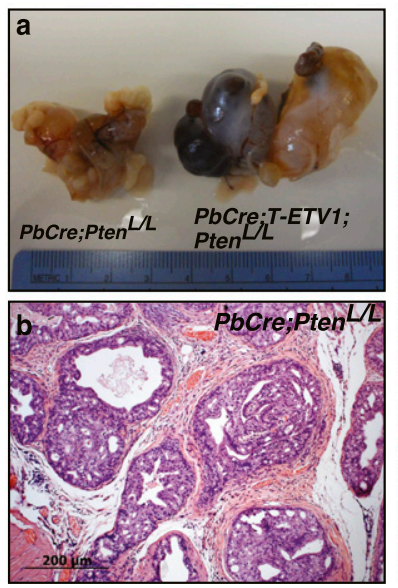
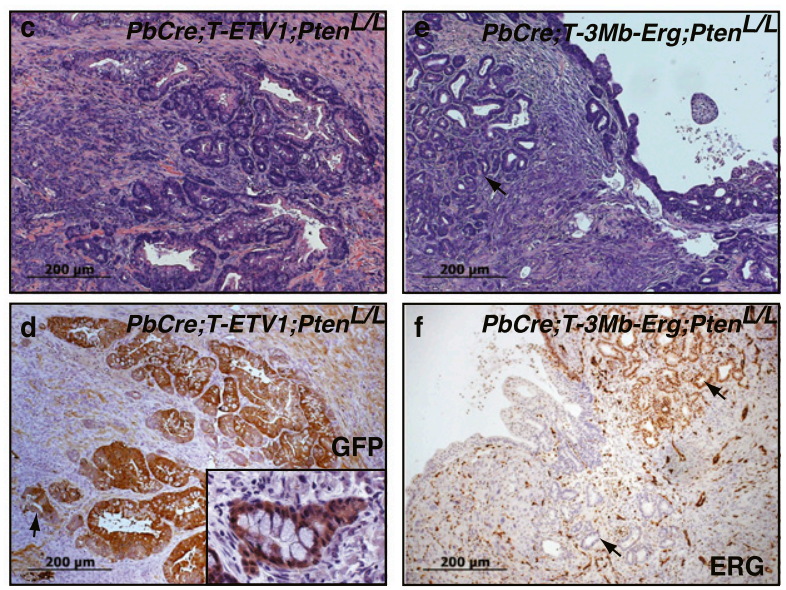

Figure 2. Cooperation of Tmprss2-ERG and Tmprss2-ETV1 gene fusions with Pten loss. (A) Bar graph summarizing histology of prostates from Pten ${ }^{+/-}$, T- $\Delta$-Erg;Pten ${ }^{+/-}$, T-ERG;Pten ${ }^{+/}$, and T-ETV1;Pten ${ }^{+/-}$males. All males were at 6-15 mo of age when checked. The youngest Pten ${ }^{+-}$, T- - -Erg;Pten ${ }^{+-}$, T-ERG;Pten ${ }^{+-}$, and T-ETV1;Pten ${ }^{+-}$males in which PIN lesions were detected were at 13, 9.5, 6.5 , and 6 mo of age, respectively. (B) IHC staining showing GFP expression (as a surrogate for ETV1 expression) in the PIN lesion of

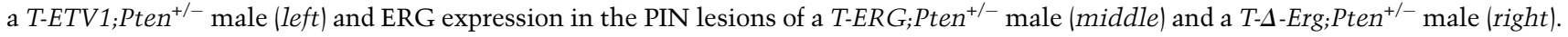
Note, in all cases, GFP or ERG staining in PIN lesions (arrows) is stronger than that in normal-appearing prostate cells. Bars, $100 \mu \mathrm{m}$. (C) Kaplan-Meier survival curve. All males were monitored for survival for at least $1 \mathrm{yr}$. The four PbCre;T-3Mb-Erg;Pten ${ }^{L / L}$ males all survived to $1 \mathrm{yr}$ and were euthanized for histology. The majority of PbCre;Pten ${ }^{L / L}$ control males were still alive even after $15 \mathrm{mo}$. Logrank test: $\left(^{\star \star}\right) P=0.0013$ for PbCre; T-ETV1;Pten ${ }^{L / L}$ in relation to PbCre;Pten ${ }^{L / L}$ controls. $(D)$ Cancer phenotypes in PbCre; Pten ${ }^{L / L}$ males with or without ETS fusions. (Panel $a$ ) Gross appearance of prostates from a 10-mo-old PbCre; T-ETV1;Pten ${ }^{L / L}$ male showing large tumor and prostatic cyst (right) and a 13-mo-old PbCre; Pten $^{L / L}$ control male (left). (Panel $b$ ) Typical localized prostate cancer seen in a control PbCre; Pten $^{L / L}$ male. (Panel $c$ ) Invasive prostate adenocarcinoma seen in a PbCre;T-ETV1;Pten ${ }^{L / L}$ male. (Panel $d$ ) GFP staining (as a surrogate for ETV1 expression) in invasive prostate adenocarcinoma cells (brown) in a PbCre;T-ETV1;Pten ${ }^{L / L}$ male (a magnified view of $\mathrm{GFP}^{+}$invasive prostate cancer cells is shown in the inset). (Panel $e$ ) Invasive prostate adenocarcinoma cells (arrow) detected in an aged $\mathrm{PbCre} ; \mathrm{T}-3 \mathrm{Mb}$-Erg; Pten $^{L / L}$ male. (Panel $f$ ) IHC staining of Erg (brown) revealing Erg ${ }^{+}$and Erg $^{-}$invasive prostate tumor cells (arrows; from the same male as in panel e) within the same section. Bars, $200 \mu \mathrm{m}$.

initially relatively weak and heterogeneous (Fig. 1C). Thus, overexpression of ETV1 or ERG correlates with the observed localized premalignant phenotype.

To test cooperation of Tmprss2-ETS with total loss of Pten, we used Pb-Cre (Wu et al. 2001) to inactivate a conditional knockout allele of Pten (Lesche et al. 2002) and generated $\mathrm{Pb}$-Cre; $\mathrm{T}-3 \mathrm{Mb}$-Erg; $\mathrm{Pten}^{L / L}$ males and $\mathrm{Pb}$-Cre; T-ETV1;Pten ${ }^{L / L}$ males. Prostate cancer development in these models was tracked by pAKT expression (Supplemental Fig. S4). Under our housing and genetic background (mixed C57/BL6-129), Pb-Cre; Pten $^{L / L}$ males developed localized PIN lesions that slowly progressed to prostate adenocarcinomas. In contrast, the Pten loss-driven prostate cancer phenotype in Pb-Cre;T-ETV1;Pten ${ }^{L / L}$ males was markedly enhanced. The majority of $\mathrm{Pb}$-Cre; T-ETV1; Pten $^{L / L}$ males died before $1 \mathrm{yr}$ of age, possibly due in part to large prostatic cyst formation (Supplemental Fig. S5). In contrast, the majority of $\mathrm{Pb}$-Cre; $\mathrm{Pten}^{L / L}$ and $\mathrm{Pb}$ Cre; T-3Mb-Erg;Pten ${ }^{L / L}$ males survived to at least $1 \mathrm{yr}$ of age (Fig. 2C). On histology, we observed aggressive GFP $^{+}$ (from the T-ETV1 allele) prostate adenocarcinoma cells invading into stroma in Pb-Cre; T-ETV1;Pten ${ }^{L / L}$ prostates (Fig. 2D, panels c,d). Prostate cancer cells metastasized locally to the urogenital area (e.g., in lymphatic vessel) 
(Supplemental Fig. S6A). In addition, we noted marked stromal responses, including sarcomatous-like lesions and regions with bone-like differentiation (Supplemental Fig. S6B). Since such lesions were negative for GFP (i.e., ETV1 expression) and pAKT (Supplemental Fig. S6C), we reasoned that they may represent a desmoplastic response in the stroma due to invasive prostate cancers developing nearby, as observed in other cancers (Dvorak 1986), rather than cancers arising from an epithelial-to-mesenchymal transition.

In younger Pb-Cre;T-3Mb-Erg;Pten ${ }^{L / L}$ males (4-7 mo), the prostate phenotype appeared indistinguishable from that of $\mathrm{Pb}$-Cre; Pten $^{L / L}$ controls. The four oldest $\mathrm{Pb}$-Cre; T-3Mb-Erg;Pten ${ }^{L / L}$ males in this cohort all survived to $1 \mathrm{yr}$ of age and were euthanized for histological examination. In these mice, we observed regions of invasive prostate adenocarcinoma not typically seen in $\mathrm{Pb}-\mathrm{Cre} ; \mathrm{Pten}^{L / L}$ control males at the same age in our cohort (Fig. 2D, panel e). However, we noted that whereas some cancer cells stained strongly for Erg expression, many were negative. In particular, we detected foci of invasive adenocarcinoma with strong Erg expression, accompanied by adjacent foci of invasive adenocarcinoma with similar histology but with little or no Erg expression (Fig. 2D, panel f). We also observed regions with invasive adenocarcinoma that were largely negative for Erg in epithelial cells (Supplemental Fig S7). The dynamic expression pattern suggests that Erg expression is up-regulated and selected for in PIN lesions under the Pten $^{+-}$- background but may not be strictly needed in invasive cancers under the total Pten loss background. In contrast, ETV1 expression appeared consistently homogeneous in invasive cancer cells, suggesting that its overexpression is required for cooperation with total Pten loss for the development of invasive adenocarcinomas (Fig. 2D, panel d).

In summary, we found that while both Tmprss2-ETV1 and Tmprss2-ERG cooperate with loss of a single copy of Pten in the development of localized prostate cancer, only Tmprss2-ETV1 appears to cooperate with full loss of Pten, leading to invasive prostate adenocarcinoma and decreased survival.

\section{ERG and ETV1 regulate distinct programs in immortalized nontumorigenic prostate cells}

The genetically engineered knock-in mice distinguished ETV1 from ERG in supporting invasive adenocarcinoma. To gain mechanistic insights into this difference, we performed an integrated genomic analysis to identify their respective target genes. First, we ectopically expressed ETV1 or ERG in immortalized human nontumorigenic prostate epithelial cells, RWPE-1 cells. Thus, RWPE-1 cells were engineered to express full-length $E R G$ or ETV1 cDNA carrying a substrate tag that permits in vivo biotinylation by coexpressed Escherichia coli biotin ligase BirA (bioERG and bioETV1) (Supplemental Fig. S8A,B). RWPE-1 cells overexpressing bioERG (R.ERG) or bioETV1 (R.ETV1) proliferated normally compared with controls (Supplemental Fig. S8C). Microarray expression profiling data strongly overlapped with those previously reported (ERG [Gupta et al. 2010] and ETV1 [Tomlins et al. 2007] in RWPE-1 cells [Supplemental Fig. S8D,E]) and yielded two findings. First, a set of genes was uniquely regulated by either ERG or ETV1. Second, both factors also regulated a common set of genes but in a largely opposing fashion (Fig. 3A). This latter observation is depicted by the quantitative two-dimensional comparison of the ERG and ETV1 data sets, which illustrates a statistically significant correlation between ERG-driven up-regulated genes and ETV1-driven down-regulated genes and vice versa (Fig. 3B). Gene ontology (GO) and Ingenuity pathway analysis (IPA) implicated genes upregulated upon ERG expression and down-regulated upon ETV1 expression that are associated with cell proliferation. In contrast, genes down-regulated on ERG expression and up-regulated by ETV1 are correlated with cell motility and lipid metabolism (Supplemental Fig. S9). As revealed by quantitative RT-PCR of select common genes, ETV1 expression induced up-regulation of genes involved in AR signaling (TMPRSS2 and SOX9) or invasion and lipid metabolism (VIMENTIN, ADRB2, and ACSL3) as well as down-regulation of cell cycle genes (E2F1 and BRCA1) (Fig. 3C). In contrast, these genes exhibited largely an opposite expression pattern in ERG-expressing RWPE-1 cells (Fig. 3C). Thus, these data point to distinct regulatory programs driven by ERG and ETV1 in nontumorigenic prostate cells.

\section{ERG and ETV1 have shared and distinct chromatin targets}

To interrogate further similarities and differences of ERG and ETV1 transcriptional programs, we performed ChIP (chromatin immunoprecipitation)-on-chip analysis. We identified ERG ChIP targets in VCaP cells (harboring TMPRSS2-ERG) by an anti-ERG antibody. As a ChIPquality antibody for ETV1 is not available, we used the bio-ChIP approach (Supplemental Fig. S10A; de Boer et al. 2003; Wang et al. 2006; Kim et al. 2008) on LNCaP cells (harboring ETV1 fusions) ectopically expressing bioETV1 and the E. coli biotin ligase BirA (Supplemental Fig. S10B). On comparison of ERG and ETV1 ChIP targets (Supplemental Table S1), we identified three subsets: ERG-ETV1 common targets, ERG-only targets, and ETV1-only targets (Fig. 4A), which are consistent with reported ChIPseq (ChIP combined with deep sequencing) data in RWPE-1 cells (Hollenhorst et al. 2011). Bound regions for each factor were typically in close proximity to the transcription start sites (TSSs) (Supplemental Fig. S10C,D). We confirmed that ERG-ETV1 common target regions were occupied by both ERG and ETV1. As anticipated, ETS-binding motifs are the most statistically significant enriched motifs within the predicted targets (Fig. 4B). Further analysis of the defined target subsets revealed clear differences of motif enrichment at common versus unique targets (Fig. 4B; Supplemental Fig. S10E), consistent with expression profiling data indicating distinct biological processes regulated by ERG and ETV1 in prostate cells (Supplemental Figs. S9A,B, S10F). 
A

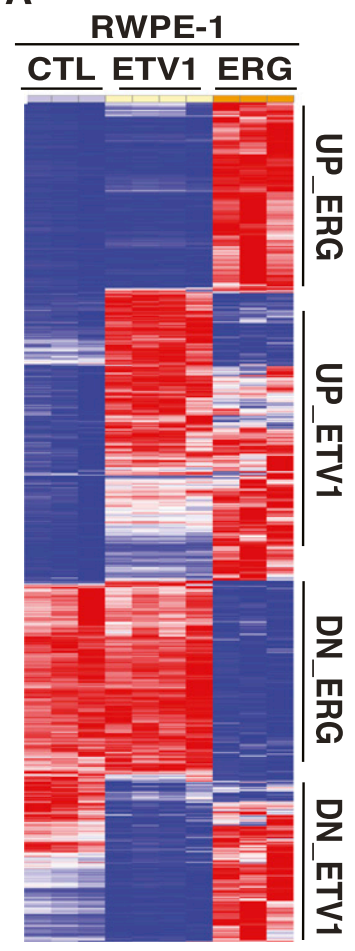

B
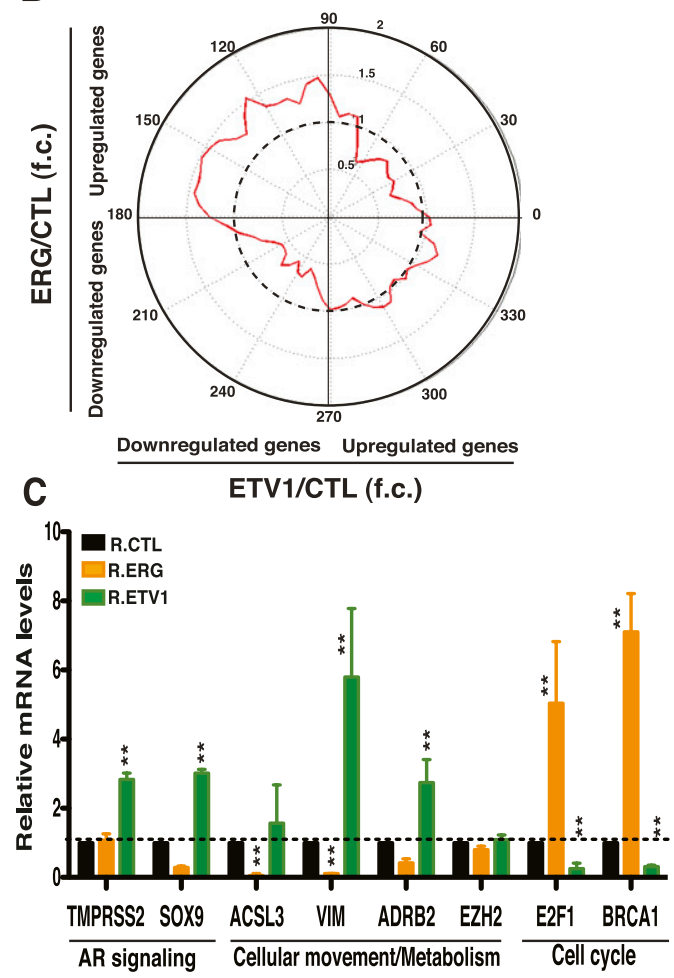

Figure 3. ERG and ETV1 regulate a common program in immortalized nontumorigenic RWPE-1 prostate cells but in an opposing fashion. (A) Expression profiling of ERG-overexpressing (R.ERG) and ETV1overexpressing (R.ETV1) RWPE-1 cells compared with BirA-expressing controls (CTL). Heat map generated by hierarchical clustering and by applying Pearson correlation and the complete linkage rule. The heat map shows differentially expressed genes (fold change, $>1.5$; false discovery rate [FDR], $<0.05$ ). (Red) Highest expression; (blue) lowest expression. (B) Bidimensional plot comparing expression profiles of genes differentially expressed (fold change, $>1.5$ ) in R.ERG versus R.CTL and in R.ETV1 versus R.CTL RWPE-1 cells. The red line represents the distribution of genes. The dotted line corresponds to a gene density fold change of 1. (C) RT-PCR analysis of select genes associated with prostate cancer pathways upon ERG or ETV1 overexpression in RWPE- 1 cells. $n=3$ per group. Error bars, $\mathrm{SEM} ;$-test: $\left({ }^{\star \star}\right) P<0.01$. If no $P$-value is indicated, $P>0.05$.
Interestingly, IPA analysis indicated that nuclear receptor signaling pathways, including those associated with estrogen, androgen, and glucocorticoid receptor signaling, were significantly enriched in ERG-ETV1 common targets (Fig. 4C). In contrast, the ERG-only subset correlated with the cell cycle network. Intriguingly, the lipid metabolism biological network as well as the Oncostatin M and IL-3 signaling pathways, which have been correlated with increased cell motility and invasiveness (Dentelli et al. 1999; Holzer et al. 2004), were enriched in the ETV1-only subset (Fig. 4C). Taken together, our combined gene expression and ChIP-on-chip analyses argue that ERG and ETV1 control distinct transcriptional programs in prostate cells.

\section{ERG and ETV1 interact differentially with the AR signaling pathway}

The AR pathway is a critical driver of tumorigenic prostate development in both androgen-dependent (AD) and castration-resistant stages (Wang et al. 2009). Our data suggest that genes associated with AR signaling belong to the ERG-ETV1 common target category (Fig. 4C; Supplemental Fig. S11A). To address potential cross-talk of ERG and ETV1 common targets with the AD program, we defined an improved androgen-driven signature from $\mathrm{AD} \mathrm{VCaP}$ and LNCaP cell lines that mitigates differences among diverse expression-based gene sets (Supplemental Fig. S12). Gene set enrichment analysis (GSEA) (Subramanian et al. 2005) revealed that this signature was significantly depleted after ETV1 knockdown in LNCaP cells (Fig. 5A) but enhanced in VCaP cells upon
ERG silencing (Fig. 5B). This finding was confirmed by quantitative RT-PCR for select AR-driven genes in LNCaP and VCaP cells (Figure 5C,D). ChIP analysis further demonstrated that, while androgen stimulated AR and ETV1 binding to the enhancers and promoters of two established AR targets, PSA and TMPRSS2, androgen decreased ERG occupancy to these AR target genes (Supplemental Fig. S11B,C). These data indicate that ETV1 cooperates with activation of AR signaling, while ERG negatively modulates the AR transcriptional program.

To determine whether ERG and ETV1 also regulate AR signaling differentially in vivo, we examined GFP expression in T-ERG and T-ETV1 males, as the reporter serves as a surrogate for the in vivo activity of the Tmprss 2 promoter in these identically engineered mice. GFP expression was readily detected in T-ETV1 prostates but barely detected in T-ERG prostates (Fig. 5E). In contrast, GFP expression was detected in estrogen receptor-positive mammary epithelial cells (Sleeman et al. 2007) of both T-ERG and T-ETV1 females (Supplemental Fig. S13). These data indicate that Tmprss 2 is indeed an estrogen and androgen dual-responsive promoter and that its promoter activity is down-regulated in T-ERG prostate cells.

To ascertain whether elevated AR signaling up-regulates the Tmprss 2 promoter activity in vivo, we bred T-ERG and T-ETV1 mice to transgenic mice that express a mutated version of AR (E231G) expressed from the $P b$ promoter $(P b-A R)$ (Han et al. 2005). In $P b-A R ; T-E R G$ double-transgenic males, we detected a strong GFP signal in the prostate (Fig. $5 \mathrm{E}$ ). The $P b-A R$ transgene is most 
A

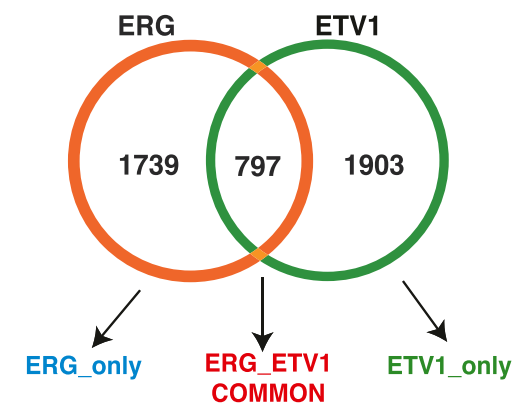

B

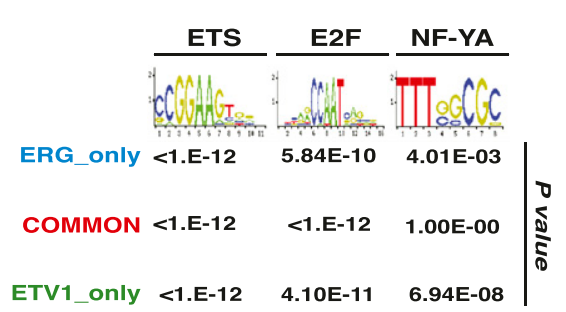

C

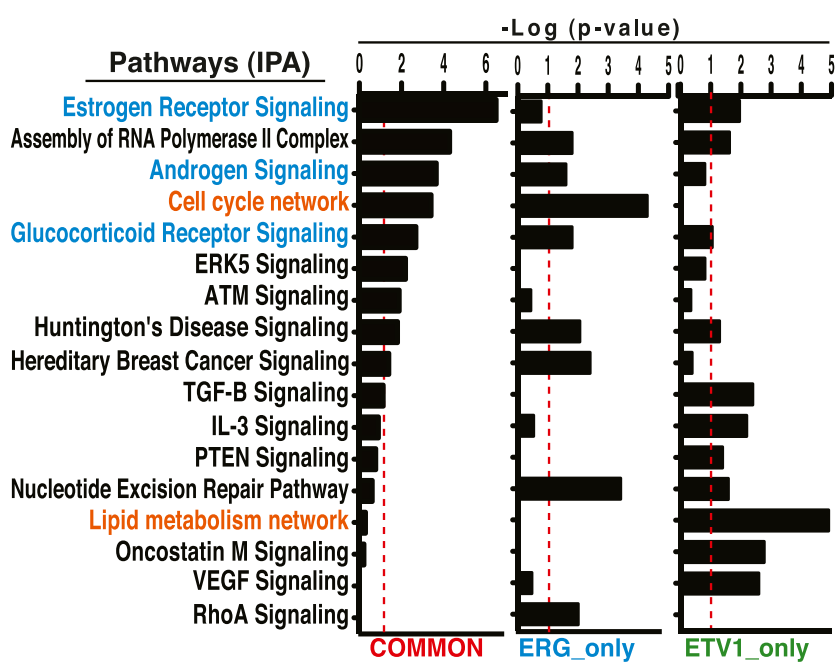

Figure 4. ERG and ETV1 drive specific transcriptional programs. (A) Venn diagram of targets occupied by ERG and ETV1. The intensity of binding at each probe was calculated by model-based analysis of tiling array (MAT) (Johnson et al. 2006). MAT scores were then normalized by quantilequantile normalization (Bolstad et al. 2003) between ETV1 and ERG ChIP-on-chip experiments. Target loci were defined as the peaks associated with $P$-value $<10^{-4}$. $(B)$ Enrichment of ETS-binding motifs and other indicated motifs in all ChIP target subsets. The Fisher exact test was applied. (C) IPA analysis of ChIP-defined target gene sets implicating common target genes in nuclear receptor signaling pathways and ETV1 unique targets in lipid metabolism network. The significance of enrichment of each gene set is shown as $-\log (P$-value $)$. active in the ventral prostate lobes (Han et al. 2005). In accord with this, we observed stronger staining for ERG in the ventral lobes of $P b-A R$; T-ERG prostates, compared with barely detectable ERG staining in T-ERG prostates (Fig. 5F). Furthermore, we measured expression levels of select AR targets in mouse prostates. In the $P b-A R$ background, most AR targets were down-regulated in T-ERG males, whereas AR targets were typically up-regulated in T-ETV1 males, thus illustrating the opposite regulation of AR signaling by ERG and ETV1 in vivo (Fig. 5G). Despite elevated AR signaling, prostates of $P b-A R$-alone as well as those of $P b-A R ; T-E R G$ and $P b-A R ; T-E T V 1$ males appeared largely normal. Taken together, human prostate cancer cell and mouse model data indicate that differential regulation of the AR pathway by ETV1 and ERG occurs not only in vitro, but, importantly, also under the physiological setting.

\section{ETV1 directs androgen metabolism in prostate epithelial cells}

In addition to the opposing regulation of common targets by ETV1 and ERG, we hypothesized that unique targets controlled by ETV1 might contribute to the aggressive phenotype seen in association with ETV1 expression. To gain mechanistic insights into programs selectively regulated by ETV1 we sorted $\mathrm{GFP}^{+}$(thus, ETV1-expressing) prostate luminal cells from T-ETV1 knock-in males and compared them with luminal cells from wild-type prostates by microarray expression profiling (Fig. 6A,B). We confirmed the luminal cell expression pattern in both sorted samples (Supplemental Fig. S14). By GSEA, we identified several cancer-associated metabolic pathways that were enriched in T-ETV1 luminal cells (Supplemental Fig. S15A). Of note, cholesterol and steroid biosynthesis pathways, both of which are strongly related to prostate tumorigenesis (Twiddy et al. 2010; Zadra et al. 2010), were most highly enriched (Fig. 6C,D). On analysis of a patient cohort with CRPCs metastatic to bone (Stanbrough et al. 2006), we observed that genes associated with the steroid hormone biosynthesis pathway and androgen and estrogen metabolism are significantly enriched in tumors with higher ETV1 expression (Supplemental Fig. S15A). HSD17B7, a gene shared by steroid biosynthesis and steroid hormone biosynthesis pathways, was up-regulated in both T-ETV1 luminal cells and ETV1high bone metastases (Supplemental Fig. S15B). HSD17B7 as well as other HSD17B enzyme genes (HSD17B4 and $H S D 17 B 10)$ are ChIP targets of ETV1 in prostate cancer cells and are components of the lipid metabolism 
A

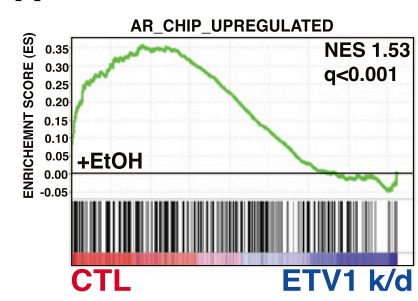

C

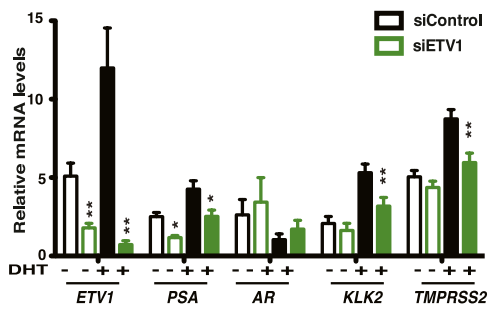

$\mathbf{F}$

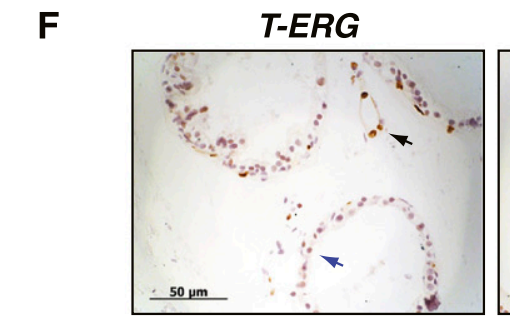

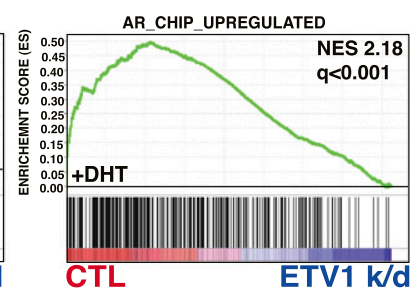

D
B

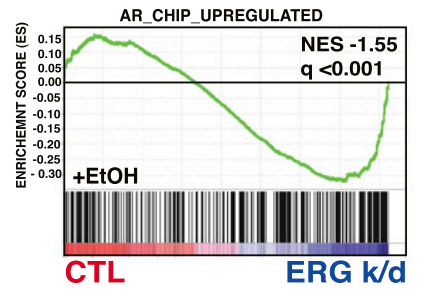

E
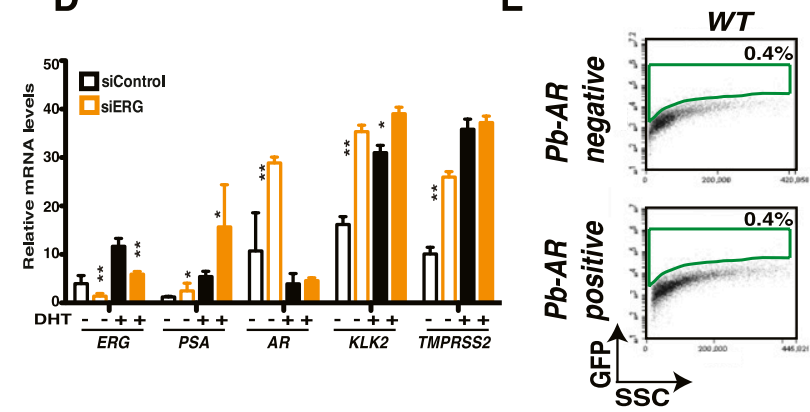

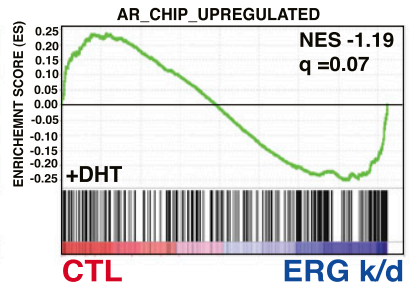

ERG k/d
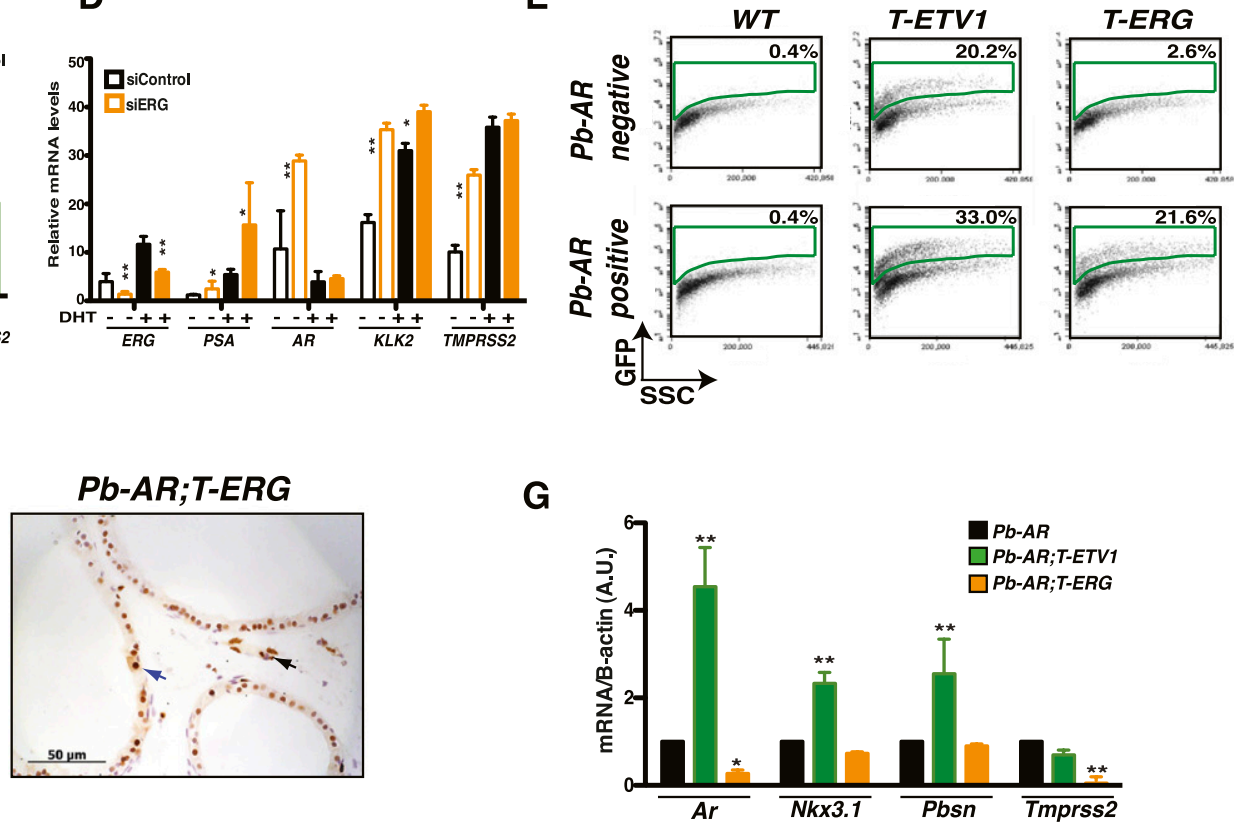

Figure 5. ERG and ETV1 regulate AR signaling in an opposite manner. (A) Androgen-induced genes are depleted in ETV1-silenced LNCaP cells upon 16-h androgen stimulation ([left] no androgen stimulation; [right] with androgen stimulation). The androgen-induced signature was obtained from the common AR ChIP targets in LNCaP and VCaP cells that were up-regulated in them upon androgen stimulation. $(B)$ Androgen-induced genes are significantly enriched in ERG-silenced VCaP cells upon 16-h androgen stimulation compared with controls ([left] no androgen stimulation; [right] with androgen stimulation). (C,D) ETV1 silencing specifically decreases expression of AR-associated genes $(C)$, whereas $E R G$ silencing increases their expression $(D)$. Mean, $n=3$; error bars, $\mathrm{SEM} ;$-test: $\left({ }^{\star}\right) P<$ $\left.0.05 ;{ }^{\star \star}\right) P<0.01$. If no $P$-value is indicated, $P>0.05$. $(E)$ Flow cytometry analysis demonstrating robust GFP ${ }^{+}$population in the T-ETV1 prostates but not in the T-ERG prostates. However, in the presence of the $P b-A R$ transgene, GFP expression can be readily detected in $P b-A R ; T-E R G$ prostates; in addition, GFP expression in $P b-A R ; T-E T V 1$ prostates appear to be further elevated. $(F)$ IHC staining showing weak ERG staining in the ventral lobe of a T-ERG knock-in male (blue arrow; compared with strong Erg staining in the endothelial cells [black arrow]) but much stronger ERG staining in the ventral lobe of a $P b-A R ; T-E R G$ male (blue arrow; almost comparable with ERG staining in endothelial cells in the same section [black arrow]). Bars, $50 \mu \mathrm{m}$. (G) Real-time PCR quantification showing up-regulation of most AR target genes in $P b-A R ; T-E T V 1$ prostates and slight down-regulation of them in $P b-A R ; T-E R G$ prostates in relation to those of $P b$ - $A R$-alone prostates. Mean, $n=3$; error bars, SEM; $t$-test: $\left({ }^{*}\right) P<0.05,\left(^{\star \star}\right) P<0.01$. If no $P$-value is indicated, $P>0.05$.

network enriched in ETV1-only ChIP targets (Fig. 4C; Supplemental Fig. S15C). By independent ChIP analysis, we validated ETV1, but not ERG, binding to the HSD17B7 and HSD17B4 promoters (Fig. 6E; Supplemental Fig. S15D). Expression of HSD17B7 in LNCaP cells was reduced upon ETV1 depletion (Fig 6F). In ETV1-overexpressing RWPE-1 cells, HSD17B7 expression trended upward (although not statistically significant) (Supplemental Fig. S16A). We also confirmed higher Hsd17b7 expression in lineage-depleted T-ETV1 prostate cells (Fig. 6G). Since HSD17B7 is critical in converting less active forms of estrogen and androgen to more active forms (Fig. 6H; Krazeisen et al. 1999), and Tmprss2 is an androgen and estrogen dual-responsive gene, we reasoned that upregulation of the steroid biosynthesis pathway by ETV1 may provide prostate cells with an intrinsic source of steroids. If this supposition were correct, such cells might be intrinsically castration-resistant. To test this prediction, T-ETV1 knock-in males as well as T-ERG and wild-type control males were castrated. We observed that almost half of prostate cells from the castrated T-ETV1 mice were $\mathrm{GFP}^{+}$. In addition, we also detected a small population of $\mathrm{GFP}^{\text {low }}$ prostate cells from the castrated T-ERG males (Supplemental Fig. S16B), consistent with a recent study and possibly reflecting the existence of a subpopulation of Tmprss $2^{+}$prostate cells that are intrinsically castration- 
A

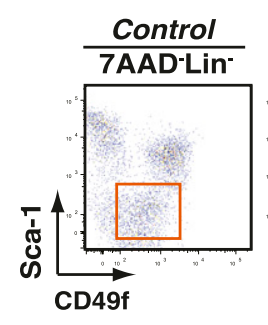

E

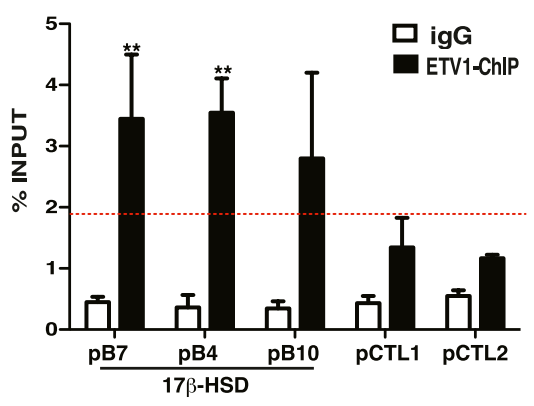

H

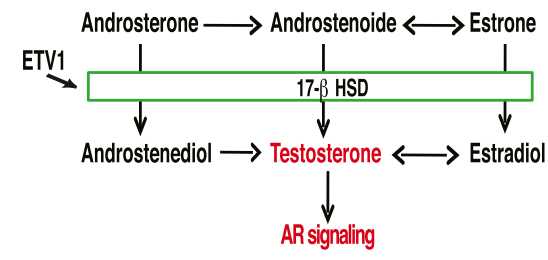

B

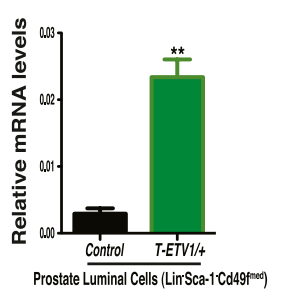

C

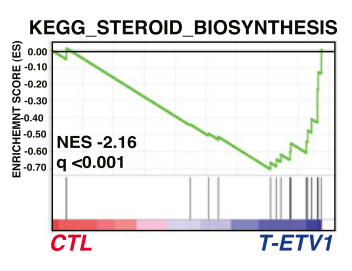

D

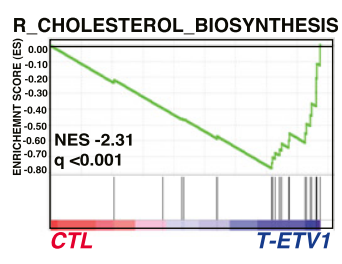

F

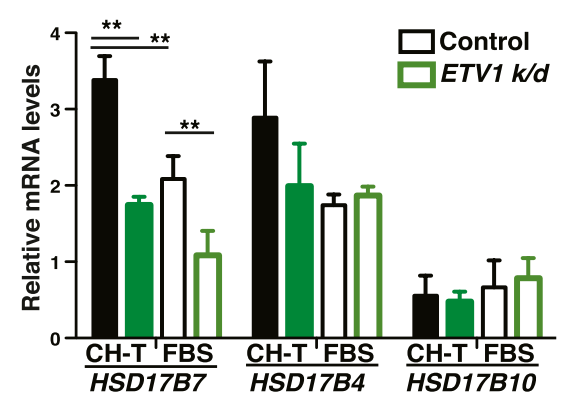

G

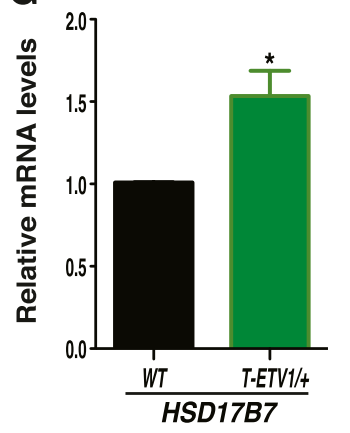

I

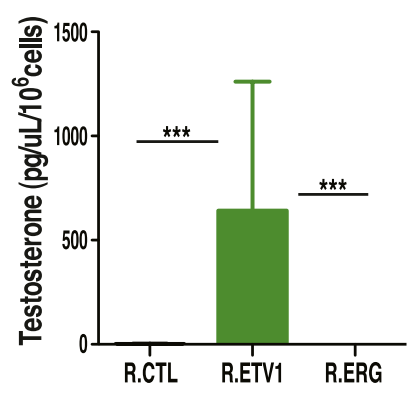

J

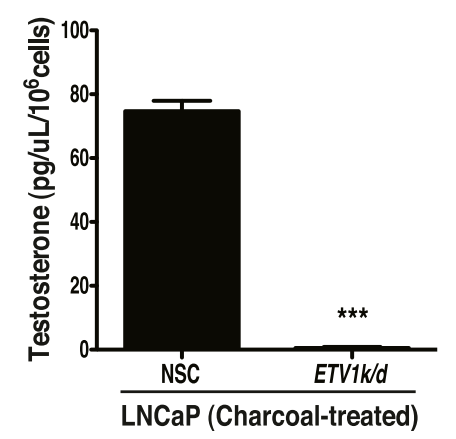

Figure 6. ETV1 regulates steroid metabolism in prostate cells. (A, right plot) Flow cytometry profiles and gating strategies showing $\mathrm{GFP}^{+}$prostate luminal cells ( $\mathrm{Lin}^{-} \mathrm{Sca}-1^{-} \mathrm{CD} 49 \mathrm{f}^{\text {med }}$ ) sorted from T-ETV1 knock-in males used for microarray analysis. (Left plot) The $\mathrm{Lin}^{-} \mathrm{Sca}-1^{-} \mathrm{CD} 49 \mathrm{f}^{\mathrm{med}}$ prostate luminal cells sorted from wild-type (WT) control males were used as the control. $(B)$ Real-time PCR quantification confirming ectopic ETV1 expression in sorted $\mathrm{GFP}^{+}$prostate luminal cells from T-ETV1 knock-in mice $($mean, $n=3$ samples per group; error bars, SEM). $(C, D)$ Steroid and cholesterol biosynthesis pathways are the top pathways significantly enriched in T-ETV1 knock-in prostate luminal cells compared with controls. Note that a critical enzyme in the steroid biosynthesis pathway, HSD17B7, is also a key enzyme in the steroid hormone biosynthesis pathway, which is enriched in metastatic prostate cancers (Supplemental Fig. S15). (E) ChIP-PCR validation of ETV1 binding to HSD17B7 (pB7), HSD17B4 (pB4), and HSD17B10 (pB10) promoters (mean, $n=5$; error bars, SEM). (pCTL1 and pCTL2) Nonspecific promoter control regions. (F) Only HSD17B7 levels significantly decreased upon knockdown of ETV1 (k/d) (mean, $n=3$, error bars, SEM) under both the androgen-deprivation condition ([CH-T] charcoal-treated) and the regular condition in the presence of serum (FBS). Conversely, HSD17B7 expression increased upon ETV1 overexpression in RPWE-1 cells (Supplemental Fig. S16A). $t$-test: $\left(^{\star \star}\right) P<0.01$. If no $P$-value is indicated, $P>0.05$. $(G) T$-ETV1 knock-in prostate cells exhibit increased $H s d 17 b 7$ expression levels compared with wild-type controls (mean, $n=3$; error bars, SEM). $t$-test: $\left({ }^{\star}\right)$ $P$-value $<0.05$. $(H)$ Schematic diagram showing the key role of 17- $\beta$ HSD enzymes, including HSD17B7, in converting androgen and estrogen from their less active forms to active forms. (I) ETV1 overexpression in RWPE-1 cells promoted the elevation of the endogenous testosterone level, while no changes were observed upon ERG overexpression (mean, $n=4$; error bars, SEM). Testosterone levels per $10^{6}$ cells (R.ETV1 mean $=642.16 \mathrm{pg} / \mu \mathrm{L}$; R.ERG mean $=0.49 \mathrm{pg} / \mu \mathrm{L} ;$ R.CTL mean $\left.=1.89 \mathrm{pg} / \mu \mathrm{L}\right) . t$-test: $\left({ }^{\star \star \star}\right) P<0.001 .(J)$ Testosterone levels were reduced in androgen-deprived (charcoal-treated) LNCaP cells upon stable ETV1 silencing (k/d) as compared with controls (mean, $n=3$; error bars, SEM). (NSC) Nonsilencing shRNA control. Testosterone levels per $10^{6}$ cells (NSC mean $=74.69$ $\mathrm{pg} / \mu \mathrm{L} ;$ R.ERG mean $=0.49 \mathrm{pg} / \mu \mathrm{L} ; E T V 1 \mathrm{k} / d$ mean $=0.56 \mathrm{pg} / \mu \mathrm{Lr}) . t$-test: $\left(^{\star \star \star}\right) P<0.001$.

resistant (Casey et al. 2012). As expected, the castrationresistant $\mathrm{GFP}^{+}$cells in T-ETV1 males exhibited higher Hsd17b7 levels than controls (Supplemental Fig. S16C).
To determine whether increased ETV1 expression enhances steroid production, we directly measured by liquid chromatography/mass spectrometry (LC/MS) the levels 
of endogenous testosterone in control, ETV1-expressing, and ERG-expressing human RWPE-1 cells. Remarkably, ETV1-expressing RWPE-1 cells showed much higher levels (>300-fold higher) of endogenous testosterone compared with ERG-expressing and control RWPE-1 cells (Fig. 6I; Supplemental Fig. S17A,B). Conversely, ETV1 knockdown in $\mathrm{LNCaP}$ cells reduced testosterone production under conditions of androgen deprivation (Fig. 6J). To further characterize this observation, we quantified the intraprostatic levels of testosterone in wild-type, T-ETV1, and T-ERG mice under noncastrated and castrated conditions. As expected, noncastrated mice showed very low concentrations of testosterone in their prostate cells. Interestingly, castrated T-ETV1 mice indeed exhibited higher testosterone levels than those of castrated wild-type and T-ERG prostate samples (Supplemental Fig. S17C). These results indicate that ETV1 expression directly regulates androgen production in prostate cells.

\section{ETV1-only gene sets associate with an aggressive phenotype in patients}

Data to this point suggest that ETV1 and its unique oncogenic program contribute to invasive prostate cancer. To relate these findings to patients, we analyzed data from a patient cohort that includes 22 primary localized and 29 metastatic samples, of which $\sim 50 \%$ carried the TMPRSS2-ERG fusion (ETV1 rearrangement status not characterized) (Stanbrough et al. 2006). In this cohort, samples exhibiting higher ERG expression correlated with localized tumors, whereas high ETV1 expression enriched for metastases (Fig. 7A). We repeated the analysis with an independent cohort of 150 prostate tumor samples from patients at Memorial Sloan-Kettering Cancer Center (MSKCC) (Taylor et al. 2010). A correlation between high ETV1 expression and metastases and the presence of more localized prostate tumors in the high $E R G$ expression group were confirmed in this second cohort (Fig. 7B). Samples with high ERG or high ETV1 expression did not overlap, consistent with a strong tendency to mutual exclusivity in both primary and metastatic samples (Supplemental Fig. S18A,B). We next interrogated whether ERG and ETV1 cooperate similarly with PTEN deletion in the MSKCC cohort by analyzing the outcome of patients carrying deletion of PTEN and overexpression of ERG or ETV1. High ERG expression with PTEN loss failed to correlate with the worse outcome (Fig. 7C). In contrast, high ETV1 expression cooperated with PTEN loss, as shown by much poorer diseasefree survival (Fig. 7D). These data are consistent with a previous report correlating greater disease recurrence with high ETV1 levels (Shin et al. 2009).

We next evaluated for the first time whether ERG- and ETV1-specific gene signatures serve as tumor biomarkers or as a predictor of aggressive behavior. We analyzed ERG and ETV1 signatures defined in our analysis of ERG and ETV1 ChIP and expression profiling data in the Swedish watchful waiting and MSKCC cohorts (Setlur et al. 2008; Taylor et al. 2010), including 362 localized prostate cancer samples and 150 patients with localized and metastatic prostate cancer, respectively. Of note, ETV1specific signatures, comprised of genes directly bound by ETV1 and up-regulated upon ETV1 expression, are associated with a high Gleason score $(>7)$ in both cohorts and with lethality in the MSKCC cohort, again highlighting a correlation between ETV1 expression and a worse disease prognosis (Fig. 7E; Supplemental Fig. S18C). Taken together, data from three independent patient cohorts concur in validating that ETV1 drives a transcriptional program in prostate cells that is distinct from that of ERG. Moreover, our data suggest for the first time that the ETV1-driven program dictates a poorer outcome in patients with prostate cancer.

\section{Discussion}

Our multidisciplinary studies reveal distinct transcription programs regulated by ERG and ETV1 in prostate cells. In particular, we show that they control overlapping gene targets but largely in an opposing fashion; they also control unique targets and pathways. Overall, the networks regulated by ERG are associated with cell cycle and DNA replication, whereas those controlled by ETV1 are related to synthesis of lipids and other metabolic pathways. These networks are distinct and contribute to different pathogenic consequences. These conclusions are validated by findings in novel knock-in mouse models and by patient outcome analysis.

\section{$A R$ signaling is a common pathway regulated by $E R G$ and ETV1 but in an opposite manner}

AR signaling is central to prostate development and tumorigenesis. Indeed, AR has recently been implicated in double-strand breaks that favor the formation of translocations involving androgen-driven promoters and ETS family members (Lin et al. 2009; Haffner et al. 2010). We observed a complex relationship between ERG- or ETV1regulated networks and AR signaling. In agreement with prior findings, we observed negative regulation of AR signaling by ERG (Yu et al. 2010). In contrast, ETV1 cooperates with AR signaling by favoring activation of the AR transcriptional program (Fig. 5). Upon androgen stimulation, ETV1 recruitment to the established PSA and TMPRSS2 regulatory elements correlates with AR binding, suggesting coordinate regulation of androgen-driven genes by ETV1 and AR.

We validated divergent regulation of AR signaling by ERG and ETV1 in vivo in knock-in mice. As Tmprss2 is an AR target, the transcriptional output from the Tmprss2 promoter serves as a reporter for AR activity. In T-ERG knock-in mice, ERG expression appears to down-regulate AR target genes, including Tmprss2, which would lead to down-regulation of its own expression, thus forming a negative regulatory loop, consistent with prior findings ( $\mathrm{Yu}$ et al. 2010). In contrast, in T-ETV1 knock-in males, ETV1 expression positively cooperated with AR signaling, leading to further enhancement of expression of AR targets, including Tmprss2, which would then support robust expression of ETV1 and the GFP reporter, reflecting a 
A

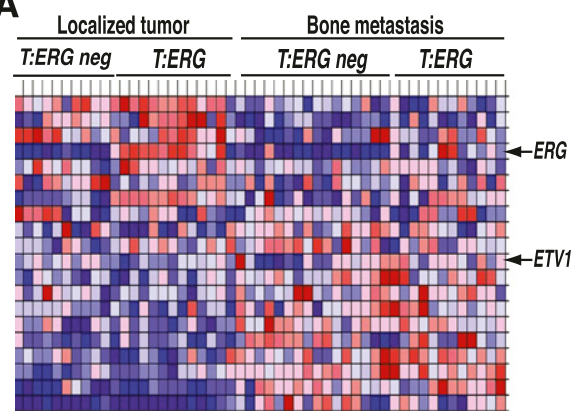

B

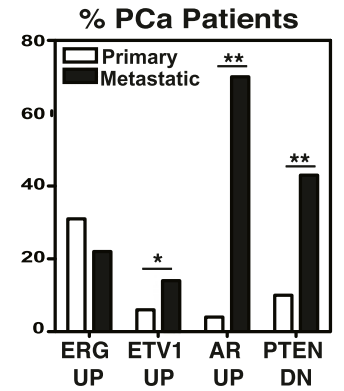

C

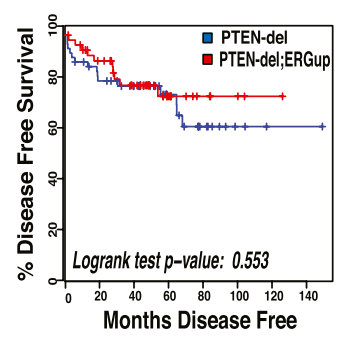

D

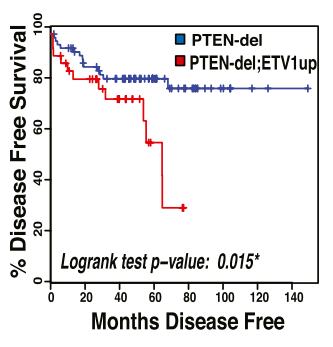

E

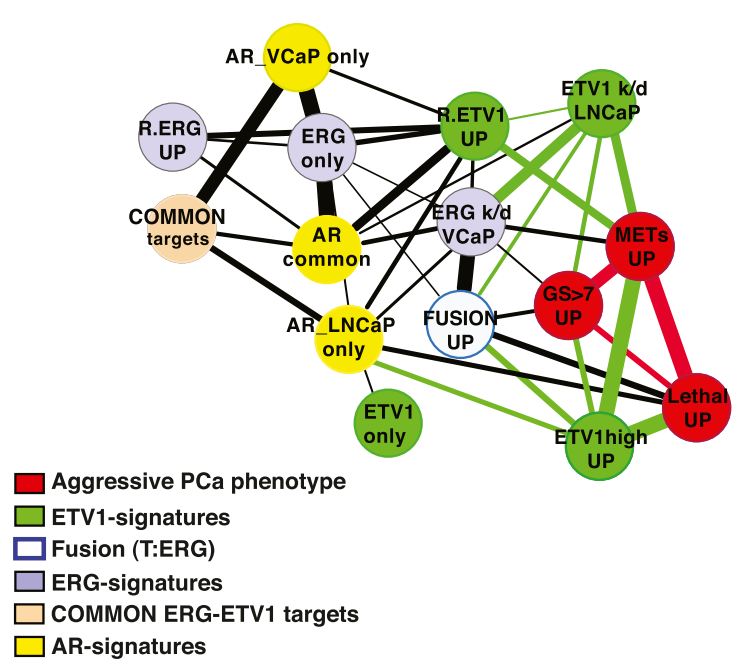

$\mathbf{F}$

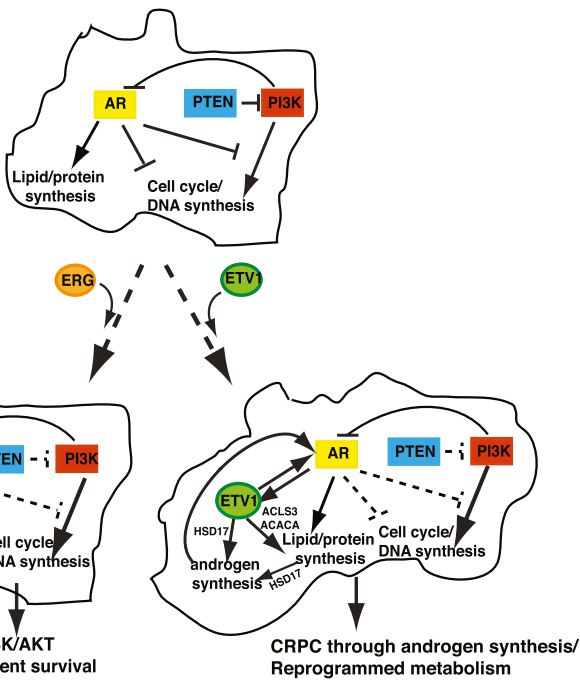

Figure 7. ETV1, rather than ERG, expression and the program it drives are associated with advanced prostate cancer in multiple patient cohorts. (A) Heat map showing ERG and ETV1 expression pattern in localized and bone metastatic prostate cancer samples using the Beth-Israel (BI) cohort data set (Stanbrough et al. 2006). Heat map generated by hierarchical clustering and by applying Pearson correlation and the complete linkage rule. Heat map showing differentially expressed select genes (fold change, $>2$; FDR, $<0.05)$. $(B)$ Graph showing ERG and ETV1 expression along prostate cancer progression from localized to metastatic samples in the MSKCC cohort (Taylor et al. 2010). The graph reveals that the number of patients carrying ETV1 overexpression (fold change, $>3$ ), PTEN deletion, and AR alterations (amplification and expression fold change, $>3$ ) increased in metastatic samples compared with localized prostate tumors, while patients carrying high levels of $E R G$ (fold change, $>3$ ) did not increase over time (also in Supplemental Fig. S18B). $t$-test: $\left(^{*}\right) P$-value $<0.05 ;\left(^{* \star}\right) P$-value $<0.01$. If no $P$-value is indicated, $P>0.05$. $(C)$ Disease-free survival plot showing that among all patients in the MSKCC cohort with PTEN deletion $(n=21)$, those with $E R G$ overexpression $(n=4)$ exhibited no survival difference from the rest of patient with PTEN deletion. $P=0.553$ by log-rank test. $(D)$ Disease-free survival plot showing that among all patients in the MSKCC cohort with PTEN deletion $(n=21)$, those with ETV1 overexpression $(n=8)$ exhibited much worse survival compared with remaining patients with PTEN deletion. $\left(^{*}\right) P=0.015$ by log-rank test. (E) Correlation between ERG- and ETV1-associated gene sets with patient prognosis. Overlap between genes enriched in patient samples associated with indolent or aggressive prostate cancer from the MSKCC cohort (Taylor et al. 2010) and ETV1 or ERG gene sets defined in Figures 3 and 4. ETV1-associated genes are enriched in patients with a higher Gleason score in the Swedish cohort (also in Supplemental Fig. S18C; Setlur et al. 2008). "UP" represents those genes up-regulated in the shown category with a fold change of $>1.5$. The significance of overlap of these gene sets was calculated by the Fisher exact test and visualized as connecting line width (cutoff, $P=0.01$ ). (Red) Aggressive prostate cancer-associated; (green) ETV1associated gene sets; (blue) TMPRSS2-ERG fusion-associated gene sets; (purple) ERG signature-associated gene sets; (yellow) ARassociated gene sets; (orange) common targets of ERG and ETV1. (F) Model illustrating the differential contribution by ERG and ETV1 to prostate tumorigenesis under the PTEN loss background. See the text for details.

positive regulatory loop. These mechanistic differences account for the striking difference in GFP intensity in the two knock-in strains, which were identically engineered (Fig. 5E). Of note, the negative loop in the T-ERG knock-in is overridden by elevated AR signaling in vivo. Indeed, the only consistent association between the TMPRSS2-ERG fusion and clinical parameters identified thus far is the association of this fusion (in particular when ERG expression was measured by IHC) with a high level of AR signaling (Minner et al. 2011; Hoogland et al. 2012). Last, in human prostate tumors with ETV1 fusions, several 5 ' fusion partners other than TMPRSS2 are also involved. 
Since the majority of these $5^{\prime}$ fusion partners are also androgen-responsive genes (Tomlins et al. 2007; Attard et al. 2008b), we reason that a similar positive regulatory loop may be operative in such tumors to enhance AR signaling and ETV1 expression. In addition, AR has been reported to regulate the endogenous ETV1 locus as well (Cai et al. 2007), suggesting a positive feedback between both the endogenous and rearranged ETV1, thus predisposing affected prostate cells for prostate cancer development.

\section{ETV1, but not ERG, reprograms metabolic pathways in normal prostate epithelial cells}

Among the unique genes up-regulated by ETV1, those encoding for enzymes involved in cholesterol and steroid biosynthesis and in cancer-associated metabolic pathways (e.g., glycolysis) (Supplemental Fig. S15A) are of particular interest. In particular, we were able to demonstrate increased production of endogenous testosterone upon ETV1 expression in both human prostate cells and T-ETV1 knock-in mice (upon castration) (Fig. 6I,J; Supplemental Fig. S17C). Due to the increased testosterone level, we anticipated observing higher levels of androstenedione or dihydrotestosterone as well; however, none were detected, possibly due to technical limitations. However, decreased levels of estrone were detected in ETV1expressing RWPE-1 cells, probably due to the promotion of testosterone synthesis (Supplemental Fig. S17B).

Although ETV1-expressing prostate cells appear ostensibly normal (Fig. 1E), their metabolic programs resemble those of cancer cells. Increased aerobic glycolysis has been observed only in advanced disease, whereas increased sterol and protein synthesis are common features of both primary and advanced prostate cancer (Swinnen et al. 2000; Rossi et al. 2003; Ettinger 2004; Bauer et al. 2005). In particular, activation of lipid metabolism has been described in most localized and metastatic prostate tumors, underscoring its potential role in prostate cancer progression (Twiddy et al. 2010; Zadra et al. 2010). Arachidonic acid metabolism and Toll-like receptor signaling inflammatory pathways (De Marzo et al. 2007) are enriched in both CRPCs with higher ETV1 expression and T-ETV1 mouse prostate cells, correlating with the extended areas of inflammation observed in T-ETV1 prostates (Fig. 1E). Thus, we speculate that this unique ETV1-controlled program, in concert with positive cooperation with AR signaling, may reprogram prostate cells for malignant progression in association with additional oncogenic events (Fig. 7F).

\section{TMPRSS2-ETV1 and TMPRSS2-ERG fusions make distinct contributions to prostate tumorigenesis}

Although both Tmprss2-ERG and Tmprss2-ETV1 cooperate with loss of a single copy of Pten in leading to localized PIN lesions, similar to those demonstrated previously in mice overexpressing ERG or ETV1 under the Probasin or viral promoters (Carver et al. 2009; King et al. 2009; Zong et al. 2009) or in mice expressing ERG from an extended human TMPRSS2 promoter (Casey et al. 2012), our mechanistic and animal model studies suggest they do so differently (Fig. 7F). Ectopic ERG expression likely represses the differentiation program of prostate cells (e.g., represses AR and AR targets and up-regulates Ezh2 and its targets, as suggested previously) (Yu et al. 2010). Interestingly, it has been reported that Pten loss leads to a castration-like phenotype by suppressing androgenresponsive gene expression through modulation of AR transcriptional activity. Moreover, conditional deletion of AR further promotes proliferation of prostate cells with Pten loss (Mulholland et al. 2011). We speculate that TMPRSS2-ERG may act in a similar fashion by downregulating $\mathrm{AR}$ and, consequently, promoting cell proliferation. Indeed, the ERG expression pattern in our murine models (Figs. 1C, 2B,D) suggests that ERG may be required primarily at early stages of the disease but may be not as functionally relevant at late stages. This may explain our observation that ERG cooperates with Pten haploinsufficiency (i.e., under a more sensitized Pten $^{+/-}$background), whereas its contribution under the total Pten loss background appears far less. The high levels of ERG expression often observed in localized fusion-positive human prostate cancers may be a secondary consequence of high activity of AR signaling in such tumors in general (i.e., similar to what we observed in $P b$-AR;T-ERG prostates) (Fig. 5E-G) rather than a critical requirement of ERG overexpression at this stage. In contrast, ectopic ETV1 expression appears to enhance androgen signaling and reprogram the metabolism of prostate cells, processes critical for both early and advanced stages of the disease. Activation of the PI3K/AKT pathway drives anabolic metabolism and tumorigenesis (Ward and Thompson 2012). We propose that TMPRSS2-ETV1 cooperates with Pten loss by further enhancing metabolic reprogramming, in particular, by favoring steroid biosynthesis, a pathway critical for invasive adenocarcinoma cells. The cooperation between ETV1 and Pten loss is also consistent with the recent finding that combined loss of Pten and COP1, a ubiquitin ligase that negatively regulates ETV1 levels, leads to more invasive prostate adenocarcinomas (Vitari et al. 2011).

In aged PbCre;T-3Mb-Erg;Pten ${ }^{L / L}$ males, we also observed invasive prostate cancer (Fig. 2E). Interestingly, in such invasive cancers, we observed a mosaic pattern of Erg expression (Fig. 2E). Several possibilities can explain this observation. Erg-expressing prostate cells may send signals to Erg-negative cells so that high levels of Erg expression are not needed in all cells of the invasive cancer. Alternatively, Erg may not be critical for the development of advanced cancer, and another genetic or epigenetic change may contribute to advanced disease. One potential genetic change is haploinsufficiency of one or more deleted genes in the interstitial region. Interestingly, ETS2, a gene residing within the interstitial region, was recently proposed to be a tumor suppressor contributing to aggressive prostate cancer cases carrying TMPRSS2ERG fusions with deletion (Grasso et al. 2012). Whether haploinsufficiency of the deleted genes (e.g., ETS2) contributes to the advanced cancer phenotype awaits further investigation. 
Distinct roles of ETV1 and ERG in prostate tumorigenesis have implications for prostate cancer therapy

Our analysis of gene expression and patient outcome data sets underscores the relevance of distinct features of ETV1regulated pathways to invasive adenocarcinoma progression. ETV1-defined, but not ERG-defined, gene sets are associated with high Gleason score and metastasis (Fig. $7 \mathrm{E}$; Supplemental Fig. S18C). Our observation that ERG expression does not correlate with the worse outcome is consistent with a recent meta-analysis describing no association of ERG with Gleason score, clinical outcome, or recurrence of the disease including 62 cohorts (Pettersson et al. 2012). Of note, ERG mRNA and protein level analysis (Markert et al. 2011; Pettersson et al. 2012) showed that TMPRSS2-ERG fusion status does not always correlate with the TMPRSS2-ERG transcriptional signature or ERG protein level in prostate cancer patients. Accordingly, most recent clinical studies have supported high ERG expression levels as a favorable prognosis biomarker (Bismar et al. 2012; Kimura et al. 2012; Suh et al. 2012). Consistent with our findings, however, ETV1 expression at the transcript level has been associated with a greater Gleason score and recurrence of the disease (Attard et al. 2008b; Shin et al. 2009). Unfortunately, thus far, it has not been possible to study clinical relevance of ETV1 at the protein level due to the lack of satisfactory antibodies. Moreover, ETV1, rather than ERG, is among AR ChIP targets defined recently from primary CRPC patient samples (Sharma et al. 2013). Last, there is also a high overlap between ETV1-associated, castration-associated, and recurrent prostate tumor signatures (Supplemental Fig. S18E). Although TMPRSS2-ETV1 fusions are only found in $\sim 1 \%-2 \%$ of all prostate cancer cases, prostate tumors with elevated ETV1 expression (5\%-10\% of all cases) are enriched in advanced disease (Fig. 7A,B), suggesting that the ETV1-driven oncogenic program may be selected for during prostate cancer progression.

In summary, our data suggest that ETV1 is a novel marker of aggressive prostate cancer, and the oncogenic program it drives may be an important therapeutic target for treating advanced prostate cancer. Metabolic enzymes (such as HSD17B7) that are regulated by ETV1 may be explored as therapeutic targets. Moreover, ETS factors have been described to modulate the Ras/MAPK pathway (Hollenhorst et al. 2011). Interestingly, ETV1 overexpression, but not that of ERG, is associated with Ras/MAPK activity in a range of tumors, including ETV1-dependent melanoma and gastrointestinal stromal tumor, where ETV1 is a master regulator of lineage (Chi et al. 2010; Jane-Valbuena et al. 2010). These observations raise the possibility that MAPK inhibitors may be explored to target ETV1-overexpressing tumors. In conclusion, our study suggests that tumors characterized by an ETV1 expression signature through either translocation or other mechanisms represent a distinct biological entity associated with aggressive prostate cancer. Future research should focus on exploring novel therapeutic approaches for this entity.

\section{Materials and methods}

\section{Mouse lines}

Tmprss2-ETS conditional knock-in mice were generated by standard gene targeting. $\mathrm{Pb}$-Cre $(\mathrm{Pb}-\mathrm{Cre} 4)$ transgenic mice were acquired from the Mouse Models of Human Cancers Consortium (MMHCC) repository. Pten conditional knockout mice $\left(\right.$ Pten $\left.^{L}\right)$ and $P b-A R$ [FVB-Tg(Pbsn-Ar ${ }^{\star}$ E231G)] transgenic mice were acquired from JAX. Pten ${ }^{+/-}$mice were generated by crossing Pten ${ }^{L /+}$ mice to Gata1-Cre mice. All studies were approved by the Institutional Animal Care and Use Committee (IACUC).

\section{Pathology, immunostaining, and flow cytometry}

Standard protocols were followed.

\section{Cell lines}

Cell lines were obtained from American Type Culture Collection and cultured accordingly. ERG or ETV1 overexpression and silencing experiments were performed by standard protocols.

\section{RT-PCR}

Real-time RT-PCR was performed according to standards protocols. All primer sequences are listed in Supplemental Table S2.

\section{Gene expression microarray analysis}

RWPE-1 stable cell clones (R.ERG, R.ETV1, and R.CTL) were grown under normal conditions. VCaP and LNCaP cells, $24 \mathrm{~h}$ after ERG or ETV1 RNAi, respectively, were grown in hormonedepleted conditions for $2 \mathrm{~d}$, and then in the presence or absence of $10 \mathrm{nM}$ DHT for $16 \mathrm{~h}$. Mouse primary prostate cells were FACS-sorted and processed according to standard procedures. Affymetrix HG133 plus 2.0 or Mouse Genome 4302.0 expression arrays were used for human or mouse samples, respectively. Gene Pattern software (Reich et al. 2006) was used for data normalization, extraction of expression values, and generation of GTC files for GSEA (Subramanian et al. 2005). A bidimensional comparison plot was used to compare differentially expressed genes $(P<0.05$ by two-tailed $t$-test $)$ in RWPE- 1 cells upon overexpression of either ETV1 or ERG.

\section{ChIP and ChIP-on-chip}

BioChIP-chip for ETV1 was performed as described (Kim et al. 2008), and conventional ChIP-chip reaction for ERG was as described (Kim et al. 2004). Affymetrix Human Promoter 1.0R array hybridization was performed at the Dana-Farber Cancer Institute Microarray Core Facility. Peak identification was calculated by MAT score (Johnson et al. 2006). For ChIP-PCR experiments, conventional ChIP reactions were performed. Antibodies used were as follows: anti-AR (N20X), anti-ERG (C17X) and antirabbit IgG from Santa Cruz Biotechnology, and anti-ETV1 kindly provided by Dr. Litovchick. The online DAVID functional annotation tool (Huang et al. 2009) and the IPA tool (Ingenuity Systems, Inc.) were used to determine the enrichment for all "FAT" GO terms and canonical pathways/networks in each gene set.

\section{Patient tumor data analysis}

Gene sets associated with indolent and aggressive prostate cancer were extracted from the Swedish, MSKCC, Sharma, and 
Glinsky cohorts (Glinsky 2004; Setlur et al. 2008; Taylor et al. 2010; Sharma et al. 2013) and analyzed for their mutual overlap between tumor cohort-derived signatures (differentially expressed genes: fold change, $>1.5$; false discovery rate [FDR], $<0.05$ ) and ERG- and ETV1-associated gene sets obtained in our studies. The overlap between gene sets was represented by a connectivity network, where the width of the connector edge was $-\log 10(P$-value $)$. The $P$-value was derived from a hypergeometric distribution by using Fisher exact test to analyze the significance of the mutual overlap. Cytoscape software version 2.8 (Cline et al. 2007) was used for the visualization of gene sets overlapping the network.

\section{Statistics}

All statistics were based on a Student's $t$-test, unless otherwise indicated. Dot plots and histograms show data means, and error bars are standard error of the mean (SEM). All statistics were performed using the data analysis package within Microsoft Excel or the analysis tool within GraphPad Prism 5.0. Kaplan-Meier survival analysis was performed using GraphPad Prism 5.0.

\section{Steroid metabolism measurement}

Steroids from RWPE-1 and androgen-deprived LNCaP cells were extracted following Lemmen et al. (2002) and quantified by LC/ MS at the Harvard FAS Center for Systems Biology.

\section{Testosterone measurement}

The intraprostatic testosterone levels were measured by a mouse testosterone ELISA kit (Calbiotech) based on the manufacturer's instructions. Briefly, mouse prostates were microdissected in cold PBS and lysed in RIPA buffer. Testosterone levels were calculated as the total amount per gram of total protein.

\section{Accession number}

The Gene Expression Omnibus accession number is GSE39388.

\section{Acknowledgments}

We thank Drs. Larisa Litovchick, Carmen Priolo, and Fatima AlShahrour for reagents, mouse surgical techniques, analysis support, and helpful discussions, and Dr. Maaike van Bragt for FACS analysis of knock-in mammary glands. This work was supported by a Career Development Award from Dana-Farber/Harvard Cancer Center Prostate Cancer SPORE (to Z.L.), a post-doctoral award from the Spanish Ministry of Science (EX2006-0958 to E.B.), and grants from the Department of Defense (PC060492 to S.H.O., PC100704 to Z.L., and PC080097 to E.B.) and NIH (U01CA105423 to S.H.O.). S.H.O. is an Investigator of Howard Hughes Medical Institute.

\section{References}

Attard G, Clark J, Ambroisine L, Fisher G, Kovacs G, Flohr P, Berney D, Foster CS, Fletcher A, Gerald WL, et al. 2008a. Duplication of the fusion of TMPRSS2 to ERG sequences identifies fatal human prostate cancer. Oncogene 27: 253-263.

Attard G, Clark J, Ambroisine L, Mills IG, Fisher G, Flohr P, Reid A, Edwards S, Kovacs G, Berney D, et al. 2008b. Heterogeneity and clinical significance of ETV1 translocations in human prostate cancer. Br J Cancer 99: 314-320.

Bauer DE, Hatzivassiliou G, Zhao F, Andreadis C, Thompson CB. 2005. ATP citrate lyase is an important component of cell growth and transformation. Oncogene 24: 6314-6322.
Bismar TA, Dolph M, Teng L-H, Liu S, Donnelly B. 2012. ERG protein expression reflects hormonal treatment response and is associated with Gleason score and prostate cancer specific mortality. Eur J Cancer 48: 538-546.

Bolstad BM, Irizarry RA, Astrand M, Speed TP. 2003. A comparison of normalization methods for high density oligonucleotide array data based on variance and bias. Bioinformatics 19: 185-193.

Cai C, Hsieh C-L, Omwancha J, Zheng Z, Chen S-Y, Baert J-L, Shemshedini L. 2007. ETV1 is a novel androgen receptorregulated gene that mediates prostate cancer cell invasion. Methods Enzymol 21: 1835-1846.

Carver BS, Tran J, Gopalan A, Chen Z, Shaikh S, Carracedo A, Alimonti A, Nardella C, Varmeh S, Scardino PT, et al. 2009. Aberrant ERG expression cooperates with loss of PTEN to promote cancer progression in the prostate. Nat Genet 41: 619-624.

Casey OM, Fang L, Hynes PG, Abou-Kheir WG, Martin PL, Tillman HS, Petrovics G, Awwad HO, Ward Y, Lake R, et al. 2012. TMPRSS2- driven ERG expression in vivo increases self-renewal and maintains expression in a castration resistant subpopulation. PLOS ONE 7: e41668.

Chi P, Chen Y, Zhang L, Guo X, Wongvipat J, Shamu T, Fletcher JA, Dewell S, Maki RG, Zheng D, et al. 2010. ETV1 is a lineage survival factor that cooperates with KIT in gastrointestinal stromal tumours. Nature 467: 849-853.

Cline MS, Smoot M, Cerami E, Kuchinsky A, Landys N, Workman C, Christmas R, Avila-Campilo I, Creech M, Gross B, et al. 2007. Integration of biological networks and gene expression data using Cytoscape. Nat Protoc 2: 23662382.

de Boer E, Rodriguez P, Bonte E, Krijgsveld J, Katsantoni E, Heck A, Grosveld F, Strouboulis J. 2003. Efficient biotinylation and single-step purification of tagged transcription factors in mammalian cells and transgenic mice. Proc Natl Acad Sci 100: $7480-7485$.

De Marzo AM, Platz EA, Sutcliffe S, Xu J, Grönberg H, Drake CG, Nakai Y, Isaacs WB, Nelson WG. 2007. Inflammation in prostate carcinogenesis. Nat Rev Cancer 7: 256-269.

Demichelis F, Fall K, Perner S, Andren O, Schmidt F, Setlur SR, Hoshida Y, Mosquera J-M, Pawitan Y, Lee C, et al. 2007. TMPRSS2:ERG gene fusion associated with lethal prostate cancer in a watchful waiting cohort. Oncogene 26: 45964599.

Dentelli P, Del Sorbo L, Rosso A, Molinar A, Garbarino G, Camussi G, Pegoraro L, Brizzi MF. 1999. Human IL-3 stimulates endothelial cell motility and promotes in vivo new vessel formation. J Immunol 163: 2151-2159.

Dvorak HF. 1986. Tumors: Wounds that do not heal. Similarities between tumor stroma generation and wound healing. $N$ Engl J Med 315: 1650-1659.

Ettinger SL. 2004. Dysregulation of sterol response elementbinding proteins and downstream effectors in prostate cancer during progression to androgen independence. Cancer Res 64: 2212-2221.

Glinsky GV. 2004. Gene expression profiling predicts clinical outcome of prostate cancer. J Clin Invest 113: 913-923.

Gopalan A, Leversha MA, Satagopan JM, Zhou Q, Al-Ahmadie HA, Fine SW, Eastham JA, Scardino PT, Scher HI, Tickoo SK, et al. 2009. TMPRSS2-ERG gene fusion is not associated with outcome in patients treated by prostatectomy. Cancer Res 69: 1400-1406.

Grasso CS, Wu Y-M, Robinson DR, Cao X, Dhanasekaran SM, Khan AP, Quist MJ, Jing X, Lonigro RJ, Brenner JC, et al. 2012. The mutational landscape of lethal castration-resistant prostate cancer. Nature 487: 239-243. 
Gupta S, Iljin K, Sara H, Mpindi JP, Mirtti T, Vainio P, Rantala J, Alanen K, Nees M, Kallioniemi O. 2010. FZD4 as a mediator of ERG oncogene-induced WNT signaling and epithelial-tomesenchymal transition in human prostate cancer cells. Cancer Res 70: 6735-6745.

Haffner MC, Aryee MJ, Toubaji A, Esopi DM, Albadine R, Gurel B, Isaacs WB, Bova GS, Liu W, Xu J, et al. 2010. Androgeninduced TOP2B-mediated double-strand breaks and prostate cancer gene rearrangements. Nat Genet 42: 668-675.

Han G, Buchanan G, Ittmann M, Harris JM, Yu X, Demayo FJ, Tilley W, Greenberg NM. 2005. Mutation of the androgen receptor causes oncogenic transformation of the prostate. Proc Natl Acad Sci 102: 1151-1156.

Hermans KG, Boormans JL, Gasi D, van Leenders GJHL, Jenster G, Verhagen PCMS, Trapman J. 2009. Overexpression of prostate-specific TMPRSS2(exon 0)-ERG fusion transcripts corresponds with favorable prognosis of prostate cancer. Clin Cancer Res 15: 6398-6403.

Hollenhorst PC, Ferris MW, Hull MA, Chae H, Kim S, Graves BJ. 2011. Oncogenic ETS proteins mimic activated RAS/ MAPK signaling in prostate cells. Genes Dev 25: 2147-2157.

Holzer RG, Ryan RE, Tommack M, Schlekeway E, Jorcyk CL. 2004. Oncostatin M stimulates the detachment of a reservoir of invasive mammary carcinoma cells: Role of cyclooxygenase-2. Clin Exp Metastasis 21: 167-176.

Hoogland AM, Jenster G, van Weerden WM, Trapman J, van der Kwast $\mathrm{T}$, Roobol MJ, Schröder FH, Wildhagen MF, van Leenders GJ. 2012. ERG immunohistochemistry is not predictive for PSA recurrence, local recurrence or overall survival after radical prostatectomy for prostate cancer. Mod Pathol 25: 471-479.

Huang DW, Sherman BT, Lempicki RA. 2009. Systematic and integrative analysis of large gene lists using DAVID bioinformatics resources. Nat Protoc 4: 44-57.

Jacquinet EE, Rao NVN, Rao GVG, Hoidal JRJ. 2000. Cloning, genomic organization, chromosomal assignment and expression of a novel mosaic serine proteinase: Epitheliasin. FEBS Lett 468: 93-100.

Jane-Valbuena J, Widlund HR, Perner S, Johnson LA, Dibner AC, Lin WM, Baker AC, Nazarian RM, Vijayendran KG, Sellers WR, et al. 2010. An oncogenic role for ETV1 in melanoma. Cancer Res 70: 2075-2084.

Johnson WE, Li W, Meyer CA, Gottardo R, Carroll JS, Brown M, Liu XS. 2006. Model-based analysis of tiling-arrays for ChIPchip. Proc Natl Acad Sci 103: 12457-12462.

Kim J, Bhinge AA, Morgan XC, Iyer VR. 2004. Mapping DNAprotein interactions in large genomes by sequence tag analysis of genomic enrichment. Nat Methods 2: 47-53.

Kim J, Chu J, Shen X, Wang J, Orkin SH. 2008. An extended transcriptional network for pluripotency of embryonic stem cells. Cell 132: 1049-1061.

Kimura T, Furusato B, Miki J, Yamamoto T, Hayashi N, Takahashi H, Kamata Y, van Leenders GJ, Visakorpi T, Egawa S. 2012. Expression of ERG oncoprotein is associated with a less aggressive tumor phenotype in Japanese prostate cancer patients. Pathol Int 62: 742-748.

King JC, Xu J, Wongvipat J, Hieronymus H, Carver BS, Leung DH, Taylor BS, Sander C, Cardiff RD, Couto SS, et al. 2009. Cooperativity of TMPRSS2-ERG with PI3-kinase pathway activation in prostate oncogenesis. Nat Genet 41: 524-526.

Klezovitch O, Risk M, Coleman I, Lucas JM, Null M, True LD, Nelson PS, Vasioukhin V. 2008. A causal role for ERG in neoplastic transformation of prostate epithelium. Proc Natl Acad Sci 105: 2105-2110.

Krazeisen A, Breitling R, Imai K, Fritz S, Möller G, Adamski J. 1999. Determination of cDNA, gene structure and chromosomal localization of the novel human $17 \beta$-hydroxysteroid dehydrogenase type 7(1). FEBS Lett 460: 373-379.

Kunderfranco P, Mello-Grand M, Cangemi R, Pellini S, Mensah A, Albertini V, Malek A, Chiorino G, Catapano CV, Carbone GM. 2010. ETS transcription factors control transcription of EZH2 and epigenetic silencing of the tumor suppressor gene Nkx3.1 in prostate cancer. PLOS ONE 5: e10547.

Lemmen JG, van den Brink CE, Legler J, van der Saag PT, van der Burg B. 2002. Detection of oestrogenic activity of steroids present during mammalian gestation using oestrogen receptor $\alpha$ - and oestrogen receptor $\beta$-specific in vitro assays. J Endocrinol 174: 435-446.

Lesche R, Groszer M, Gao J, Wang Y, Messing A, Sun H, Liu X, Wu H. 2002. Cre/loxP-mediated inactivation of the murine Pten tumor suppressor gene. Genesis 32: 148-149.

Lin C, Yang L, Tanasa B, Hutt K, Ju B-G, Ohgi KA, Zhang J, Rose DW, Fu X-D, Glass CK, et al. 2009. Nuclear receptor-induced chromosomal proximity and DNA breaks underlie specific translocations in cancer. Cell 139: 1069-1083.

Markert EK, Mizuno H, Vazquez A, Levine AJ. 2011. Molecular classification of prostate cancer using curated expression signatures. Proc Natl Acad Sci 108: 21276-21281.

Minner S, Enodien M, Sirma H, Luebke AM, Krohn A, Mayer PS, Simon R, Tennstedt P, Muller J, Scholz L, et al. 2011. ERG status is unrelated to PSA recurrence in radically operated prostate cancer in the absence of antihormonal therapy. Clin Cancer Res 17: 5878-5888.

Mulholland DI, Tran LM, Li Y, Cai H, Morim A, Wang S, Plaisier S, Garraway IP, Huang J, Graeber TG, et al. 2011. Cell autonomous role of PTEN in regulating castration-resistant prostate cancer growth. Cancer Cell 19: 792-804.

Nam RK, Sugar L, Yang W, Srivastava S, Klotz LH, Yang L-Y, Stanimirovic A, Encioiu E, Neill M, Loblaw DA, et al. 2007. Expression of the TMPRSS2:ERG fusion gene predicts cancer recurrence after surgery for localised prostate cancer. $\mathrm{Br} J$ Cancer 97: 1690-1695.

Oikawa T, Yamada T. 2003. Molecular biology of the Ets family of transcription factors. Gene 303: 11-34.

Pettersson A, Graff RE, Bauer SR, Pitt MJ, Lis RT, Stack EC, Martin NE, Kunz L, Penney KL, Ligon AH, et al. 2012. The TMPRSS2:ERG rearrangement, ERG expression, and prostate cancer outcomes: A cohort study and meta-analysis. Cancer Epidemiol Biomarkers Prev 21: 1497-1509.

Reich M, Liefeld T, Gould J, Lerner J, Tamayo P, Mesirov JP. 2006. GenePattern 2.0. Nat Genet 38: 500-501.

Rossi S, Graner E, Febbo P, Weinstein L, Bhattacharya N, Onody T, Bubley G, Balk S, Loda M. 2003. Fatty acid synthase expression defines distinct molecular signatures in prostate cancer. Mol Cancer Res 1: 707-715.

Seth A, Watson DK. 2005. ETS transcription factors and their emerging roles in human cancer. Eur J Cancer 41: 2462-2478.

Setlur SR, Mertz KD, Hoshida Y, Demichelis F, Lupien M, Perner S, Sboner A, Pawitan Y, Andrén O, Johnson LA, et al. 2008. Estrogen-dependent signaling in a molecularly distinct subclass of aggressive prostate cancer. I Natl Cancer Inst 100: 815-825.

Sharma NL, Massie CE, Ramos-Montoya A, Zecchini V, Scott HE, Lamb AD, Macarthur S, Stark R, Warren AY, Mills IG, et al. 2013. The androgen receptor induces a distinct transcriptional program in castration-resistant prostate cancer in man. Cancer Cell 23: 35-47.

Shin S, Kim T-D, Jin F, Van Deursen JM, Dehm SM, Tindall DJ, Grande JP, Munz J-M, Vasmatzis G, Janknecht R. 2009. Induction of prostatic intraepithelial neoplasia and modulation of androgen receptor by ETS variant 1/ETS-related protein 81. Cancer Res 69: 8102-8110. 
Sleeman KE, Kendrick H, Robertson D, Isacke CM, Ashworth A, Smalley MJ. 2007. Dissociation of estrogen receptor expression and in vivo stem cell activity in the mammary gland. J Cell Biol 176: 19-26.

Stanbrough M, Bubley GJ, Ross K, Golub TR, Rubin MA, Penning TM, Febbo PG, Balk SP. 2006. Increased expression of genes converting adrenal androgens to testosterone in androgen-independent prostate cancer. Cancer Res 66: 2815 2825.

Subramanian A, Tamayo P, Mootha VK, Mukherjee S, Ebert BL, Gillette MA, Paulovich A, Pomeroy SL, Golub TR, Lander ES, et al. 2005. Gene set enrichment analysis: A knowledgebased approach for interpreting genome-wide expression profiles. Proc Natl Acad Sci 102: 15545-15550.

Suh JH, Park J-W, Lee C, Moon KC. 2012. ERG immunohistochemistry and clinicopathologic characteristics in Korean prostate adenocarcinoma patients. Korean J Pathol 46: 423 428.

Swinnen JV, Vanderhoydonc F, Elgamal AA, Eelen M, Vercaeren I, Joniau S, Van Poppel H, Baert L, Goossens K, Heyns W, et al. 2000. Selective activation of the fatty acid synthesis pathway in human prostate cancer. Int J Cancer 88: 176-179.

Taylor BS, Schultz N, Hieronymus H, Gopalan A, Xiao Y, Carver BS, Arora VK, Kaushik P, Cerami E, Reva B, et al. 2010. Integrative genomic profiling of human prostate cancer. Cancer Cell 18: 11-22.

Tomlins SA, Rhodes DR, Perner S, Dhanasekaran SM, Mehra R, Sun X-W, Varambally S, Cao X, Tchinda J, Kuefer R, et al. 2005. Recurrent fusion of TMPRSS2 and ETS transcription factor genes in prostate cancer. Science 310: 644-648.

Tomlins SA, Laxman B, Dhanasekaran SM, Helgeson BE, Cao X, Morris DS, Menon A, Jing X, Cao Q, Han B, et al. 2007. Distinct classes of chromosomal rearrangements create oncogenic ETS gene fusions in prostate cancer. Nature 448: 595-599.

Tomlins SA, Laxman B, Varambally S, Cao X, Yu J, Helgeson BE, Cao Q, Prensner JR, Rubin MA, Shah RB, et al. 2008. Role of the TMPRSS2-ERG gene fusion in prostate cancer. Neoplasia 10: 177-188.

Twiddy AL, Leon CG, Wasan KM. 2010. Cholesterol as a potential target for castration-resistant prostate cancer. Pharm Res 28: $423-437$.

Vitari AC, Leong KG, Newton K, Yee C, O’Rourke K, Liu J, Phu L, Vij R, Ferrando R, Couto SS, et al. 2011. COP1 is a tumour suppressor that causes degradation of ETS transcription factors. Nature 474: 403-406.

Wang J, Rao S, Chu J, Shen X, Levasseur DN, Theunissen TW, Orkin SH. 2006. A protein interaction network for pluripotency of embryonic stem cells. Nature 444: 364-368.

Wang Q, Li W, Zhang Y, Yuan X, Xu K, Yu J, Chen Z, Beroukhim R, Wang H, Lupien M, et al. 2009. Androgen receptor regulates a distinct transcription program in androgen-independent prostate cancer. Cell 138: 245-256.

Ward PS, Thompson CB. 2012. Metabolic reprogramming: A cancer hallmark even warburg did not anticipate. Cancer Cell 21: 297-308.

Wei G-H, Badis G, Berger MF, Kivioja T, Palin K, Enge M, Bonke M, Jolma A, Varjosalo M, Gehrke AR, et al. 2010. Genomewide analysis of ETS-family DNA-binding in vitro and in vivo. EMBO J 29: 2147-2160.

Wilt TJ, Brawer MK, Jones KM, Barry MJ, Aronson WJ, Fox S, Gingrich JR, Wei JT, Gilhooly P, Grob BM, et al. 2012. Radical prostatectomy versus observation for localized prostate cancer. $N$ Engl I Med 367: 203-213.

Wu X, Wu J, Huang J, Powell WC, Zhang JF, Matusik RJ, Sangiorgi FO, Maxson RE, Sucov HM, Roy-Burman P. 2001.
Generation of a prostate epithelial cell-specific Cre transgenic mouse model for tissue-specific gene ablation. Mech Dev 101: 61-69.

Yu J, Yu J, Mani R-S, Cao Q, Brenner CJ, Cao X, Wang X, Wu L, Li J, Hu M, et al. 2010. An integrated network of androgen receptor, polycomb, and TMPRSS2-ERG gene fusions in prostate cancer progression. Cancer Cell 17: 443-454.

Zadra G, Priolo C, Patnaik A, Loda M. 2010. New strategies in prostate cancer: Targeting lipogenic pathways and the energy sensor AMPK. Clin Cancer Res 16: 3322-3328.

Zong Y, Xin L, Goldstein AS, Lawson DA, Teitell MA, Witte ON. 2009. ETS family transcription factors collaborate with alternative signaling pathways to induce carcinoma from adult murine prostate cells. Proc Natl Acad Sci 106: 1246512470. 


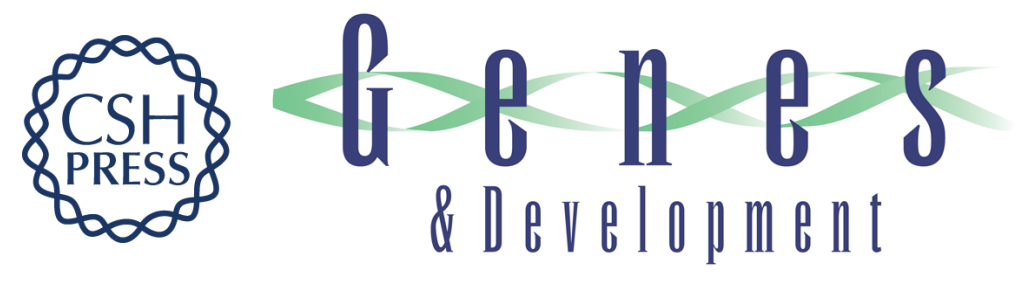

\section{ETV1 directs androgen metabolism and confers aggressive prostate cancer in targeted mice and patients}

Esther Baena, Zhen Shao, Douglas E. Linn, et al.

Genes Dev. 2013, 27:

Access the most recent version at doi:10.1101/gad.211011.112

Supplemental http://genesdev.cshlp.org/content/suppl/2013/03/19/27.6.683.DC1
Material

References This article cites 72 articles, 23 of which can be accessed free at: http://genesdev.cshlp.org/content/27/6/683.full.html\#ref-list-1

License Freely available online through the Genes \& Development Open Access option.

Email Alerting Receive free email alerts when new articles cite this article - sign up in the box at the top Service right corner of the article or click here.

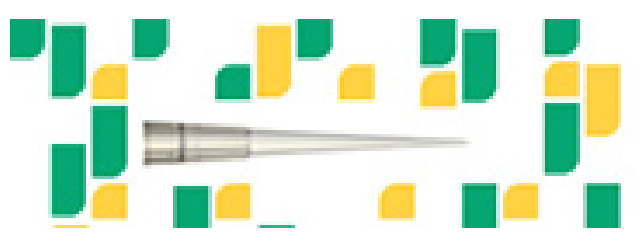

Focused on your science. 